\title{
Singularities of vector fields on $\mathbb{R}^{3}$ determined by their first non-vanishing jet
}

\author{
PATRICK BONCKAERT, $\dagger$ FREDDY DUMORTIER $†$ \\ AND SEBASTIAN VAN STRIEN \\ $†$ Limburgs Universitair Centrum, B-3610 Diepenbeek, Belgium \\ $\ddagger$ Math. Dept., T.U. Delft, PO Box 356, 2600 AJ Delft, The Netherlands
}

(Received January 1987 and revised July 1988)

\begin{abstract}
In this paper we will present a result which gives a sufficient condition for a vector field $X$ on $\mathbb{R}^{3}$ to be equivalent at a singularity to the first non-vanishing jet $j^{k} X(p)$ of $X$ at $p$. This condition - which only depends on the homogeneous vector field defined by $j^{k} X(p)$ - is stated in terms of the blown-up vector field $\bar{X}$ (which is defined on $S^{2} \times \mathbb{R}$ ), and essentially means that there are no saddleconnections for $\bar{X} \mid S^{2} \times\{0\}$.

The key tool in the proof will be a result of local normal linearization along a codimension 1 submanifold $M$ providing a $C^{0}$ conjugacy having a normal derivative along $M$ equal to 1 .
\end{abstract}

\section{Introduction and statement of results}

Let $X$ be a $C^{p}$ vector field on $\mathbb{R}^{3}$ with a singularity at $0, X(0)=0, p \geq 3$. Assume that $X$ is non-flat, i.e. that one of the jets of $X$ at 0 is non-zero. Let $k$ be such that $j_{k} X(0) \neq 0$ and $j_{k-1} X(0)=0(k \leq p-2)$. We call the homogeneous polynomial vector field $X_{k}=j_{k} X(0)$ the first-non-vanishing jet of $X$ at 0 .

We also need to define the blowing-up of a vector field. Let $S^{2}$ be the unit sphere in $\mathbb{R}^{3}$ and let $\Phi: S^{2} \times \mathbb{R} \rightarrow \mathbb{R}^{3}$ be defined by $(u, r) \rightarrow r \cdot u$ (the spherical coordinates).

Define $\tilde{X}$ by $\Phi_{*} \tilde{X}=X$ and let

$$
\bar{X}=\frac{1}{r^{k-1}} \tilde{X}
$$

where $k$ is as before. $\bar{X}$ is a $C^{p-k}$ vector field on $S^{2} \times \mathbb{R}$.

Finally we say that two vector fields $X$ and $Y$ are $C^{0}$-equivalent in 0 if there is a homeomorphism on a neighbourhood of 0 onto its image, mapping orbits of $X$ onto orbits of $Y$ preserving the orientation of orbits. If the homeomorphism also preserves the parametrization we call it a $C^{0}$-conjugacy.

TheOREM 1. Let $X$ be a $C^{p}$ vector field, $p \geq 3$, and $X_{k}$ be its first non-vanishing jet $(k \leq p-2)$. Furthermore let $\bar{X}$ be the blown-up vector field associated with $X$ at 0. Assume that

(1) $\bar{X} \mid S^{2} \times\{0\}$ is Morse-Smale.

(2) All singularities and periodic orbits of $\bar{X}$ are hyperbolic (in $S^{2} \times \mathbb{R}$ ). Then $\bar{X}$ is 
$C^{0}$-equivalent to $\bar{X}_{k}$ near $S^{2} \times\{0\}$ and a fortiori $X$ is locally $C^{0}$-equivalent to $X_{k}$ near 0 .

The proof of the theorem occupies the main body of the paper (i.e. $\S 2$ ). It relies on two 'technical' theorems, which we have put in 2 separate appendices. These theorems concern local normal linearizability along a codimension-one invariant submanifold and are valid in all dimensions. As we will see, it is not important that the critical elements of $\bar{X} \mid S^{2} \times\{0\}$ are hyperbolic, but it is certainly essential that there are no 'saddle-connections', see [Str].

If in addition $\bar{X} \mid S^{2} \times\{0\}$ has no periodic orbits, our proof will imply that $\bar{X}$ and $\bar{X}_{k}$ are $C^{0}$-conjugate in the neighbourhood of $S^{2} \times\{0\}$. Of course this induces also a $C^{0}$-conjugacy between $X / r^{k-1}$ and $X_{k} / r^{k-1}$.

The conditions (1) and (2) do not imply that $\bar{X}$ is structurally stable, only that $\bar{X} \mid S^{2} \times\{0\}$ is structurally stable. This is because $\bar{X}$ may have two periodic orbits $\gamma_{1}$ and $\gamma_{2}$ on $S^{2} \times\{0\}$ both of saddle-type of $\bar{X}$ such that $W^{u}\left(\gamma_{1}\right) \cap\left(S^{2} \times\{0\}\right)$ and $W^{s}\left(\gamma_{2}\right) \cap\left(S^{2} \times\{0\}\right)$ have an intersection. Then $W^{u}\left(\gamma_{1}\right)$ and $W^{s}\left(\gamma_{2}\right)$ have a nontransversal intersection. In [ST] it is shown that moduli of stability can appear for homogeneous vector fields with two limit cycles. We have evidence to conjecture that it is even possible to give such an example where (1) and (2) are satisfied. Of course, it follows from our theorem that the space of moduli of stability is finite dimensional.

The theorem was shown before in special cases. Shafer in [Sh] proved it if $X$ is a gradient vector field. Camacho in [C2] generalized this to the case where $\bar{X} \mid S^{2} \times\{0\}$ has no periodic orbits. Urbina et al. in [ULL] treated the case where $\bar{X} \mid S^{2} \times\{0\}$ has no orbits connecting limit cycles of saddle type. In these three papers, the authors also prove the stability of their vector fields inside the space of all vector fields with $j_{k-1} X(0)=0$. In particular, the homogeneous vector fields they consider are stable in the space of homogeneous vector fields of degree $k$. Camacho proved in [C1] that, for $k=2$, it is also a generic condition for a homogeneous vector field of degree $k$ to fulfill conditions (1) and (2) in our theorem. Recently, Camacho has announced that she has proven the same result for $k>2$.

To end let us remark that conditions (1) and (2) are equivalent to (1) and (2') with $\left(2^{\prime}\right): X_{k}$ has no singularities or periodic orbits in the neighbourhood of 0 , (apart from the singularity 0 ).

Some properties of blowing up of singularities. To simplify things we assume that $X(x)$ is defined for all $x \in \mathbb{R}^{3}$. This is no restriction. It should be noted that everything in this section holds in $\mathbb{R}^{n}$ for arbitrary $n$. Throughout this paper we will denote the flow of a vector field $Y$ by $\phi_{Y}(t, x)$.

Property 1. $\bar{X}$ is a $C^{p-k}$ vector field, in particular it is at least $C^{2}$.

Property 2. Let $(u, r)$ denote coordinates on $S^{2} \times \mathbb{R}$. If $X$ and $Y$ have the same $k$-jet in 0 (always assuming $j_{k-1} X(0)=j_{k-1} Y(0)=0$ ) and if we write

$$
\bar{X}=\bar{X}_{T} \frac{\partial}{\partial u}+\bar{X}_{N} \frac{\partial}{\partial r} \quad \text { and } \quad \bar{Y}_{T} \frac{\partial}{\partial u}+\bar{Y}_{N} \frac{\partial}{\partial r}
$$


then there exist $C^{p-k-1}$ resp. $C^{p-k-2}$ functions $f_{T}: S^{2} \times \mathbb{R} \rightarrow \mathbb{R}^{2}$ resp. $f_{N}: S^{2} \times \mathbb{R} \rightarrow \mathbb{R}$ such that $\bar{X}_{T}-\bar{Y}_{T}=r f_{T}$ and $\bar{X}_{N}-\bar{Y}_{N}=r^{2} f_{N}$.

Proof. Let $B: \mathbb{R}^{3} \backslash\{0\} \rightarrow S^{2} \times \mathbb{R}: x \rightarrow(x /|x|,|x|)$ be the inverse of $\Phi$. Then $B_{*} X=\tilde{X}$. A straightforward calculation yields:

so for $u=x /|x|$ and $r=|x|$ we get:

$$
D B(x)=\left(\frac{|x| \cdot-\left\langle\frac{x}{|x|}, \cdot\right\rangle x}{|x|^{2}},\left\langle\frac{x}{|x|}, \cdot\right\rangle\right)
$$

$$
\begin{aligned}
\tilde{X}(u, r) & =D B(x) \cdot X(x) \\
& =\left(r^{-1}(X(r u)-\langle u, X(r u)\rangle u),\langle u, X(r u)\rangle\right) .
\end{aligned}
$$

We can write $X=Y+O\left(|x|^{k+1}\right)$. So if we divide $\tilde{X}$ and $\tilde{Y}$ by $r^{k-1}$ we get:

$$
\begin{aligned}
& \bar{X}_{T}(u, r)-\bar{Y}_{T}(u, r)=\frac{1}{r^{k}} O\left(|r u|^{k+1}\right) . \\
& \bar{X}_{N}(u, r)-\bar{Y}_{N}(u, r)=\frac{1}{r^{k-1}} O\left(|r u|^{k+1}\right) .
\end{aligned}
$$

This proves property 2 .

Corollary 1. $\bar{X} \mid S^{2} \times\{0\}$ is completely determined by $j_{k} X(0)$. So $\bar{X}$ and $\bar{X}_{k}$ coincide on $S^{2} \times\{0\}$.

Corollary 2. (a) Let $p \in S^{2} \times\{0\}$ be a singularity of $\bar{X}$. The eigenvalues of $D \bar{X}(p)$ (considered in $S^{2} \times \mathbb{R}$ ) only depend on $j_{k} X(0)$. (b) Let $\gamma \subset S^{2} \times\{0\}$ be a periodic orbit of $\bar{X}$ and let $P$ be a Poincaré map for $\gamma$. The eigenvalues of $P$ only depend on $j_{k} X(0)$.

Proof. (a) This follows directly from Property 2. (b) Fix a point $p \in \gamma$. Then $\Sigma=$ $\left\{q \in S^{2} \times \mathbb{R} \mid\langle q-p, \bar{X}(p)\rangle=0\right\}$ is a local section of $\gamma$ which we use for defining the Poincaré map $P$. Let $\phi_{\bar{X}}$ denote the flow of $\bar{X}$; consider the following equation in $(t, q) \in \mathbb{R} \times \Sigma:$

$$
F(t, q)=\left\langle\phi_{\bar{X}}(t, q)-p, \bar{X}(p)\right\rangle=0 .
$$

Let $t(q)$ be a $C^{\infty}$ function with $F(t(q), q)=0$ such that $P(q)=\phi_{\bar{X}}(t(q), q)$; put $t(p)=T_{0}$.

We write $\phi_{\bar{X}}=\left(\phi_{\bar{X}}^{\mathrm{T}}, \phi_{\bar{X}}^{\mathrm{N}}\right) \in S^{2} \times \mathbb{R}$ and $P=\left(P^{\mathrm{T}}, P^{\mathrm{N}}\right)$. As $S^{2} \times\{0\}$ is invariant for $\bar{X}$ we have $D_{u} P^{\mathrm{N}}(p)=0$.

Because of Property $2, D_{u} P^{\mathrm{T}}(p)$ only depends on $j_{k} X(0)$. So the only thing to check is that $\left(\partial P^{\mathrm{N}} / \partial r\right)(p)$ only depends on $j_{k} X(0)$. We have

$$
\begin{aligned}
\frac{\partial P^{\mathrm{N}}}{\partial r}(p) & =\frac{\partial \phi_{\bar{X}}^{\mathrm{N}}}{\partial t}\left(T_{0}, p\right) \frac{\partial t}{\partial r}(p)+\frac{\partial \phi_{\bar{X}}^{\mathrm{N}}}{\partial r}\left(T_{0}, p\right) \\
& =\bar{X}_{\mathrm{N}}\left(\phi_{\bar{X}}\left(T_{0}, p\right)\right) \cdot \frac{\partial t}{\partial r}(p)+\frac{\partial \phi_{\bar{X}}^{\mathrm{N}}}{\partial r}\left(T_{0}, p\right) \\
& =\bar{X}_{\mathrm{N}}(p) \frac{\partial t}{\partial r}(p)+\frac{\partial \phi_{\bar{X}}^{\mathrm{N}}}{\partial r}\left(T_{0}, p\right) \\
& =\frac{\partial \phi_{\bar{X}}^{\mathrm{N}}}{\partial r}\left(T_{0}, p\right) .
\end{aligned}
$$


The variational equation gives:

$$
\left\{\begin{array}{l}
\frac{\partial}{\partial t}\left(D_{(u, r)} \phi_{\bar{X}}\right)(t, p)=D \bar{X}\left(\phi_{\bar{X}}(t, p)\right) \circ D_{(u, r)} \phi_{\bar{X}}(t, p) \\
D_{(u, r)} \phi_{\bar{X}}(0, p)=\text { Identity }
\end{array}\right.
$$

where $(u, r)$ represent coordinates on $S^{2} \times \mathbb{R}$.

If we write $D_{(u, r)} \phi_{\bar{X}}(t, p)=U(t)$ this gives, with respect to the product $S^{2} \times \mathbb{R}$ :

$$
\left[\begin{array}{ll}
U_{\mathrm{TT}} & U_{\mathrm{TN}} \\
U_{\mathrm{NT}} & U_{\mathrm{NN}}
\end{array}\right]^{\prime}=\left[\begin{array}{cc}
D_{u} \bar{X}^{\mathrm{T}}\left(\phi_{\bar{X}}(t, p)\right) & \frac{\partial \bar{X}^{\mathrm{T}}}{\partial r}\left(\phi_{\bar{X}}(t, p)\right) \\
0 & \frac{\partial \bar{X}^{\mathrm{N}}}{\partial r}\left(\phi_{\bar{X}}(t, p)\right)
\end{array}\right]\left[\begin{array}{cc}
U_{\mathrm{TT}} & U_{\mathrm{TN}} \\
U_{\mathrm{NT}} & U_{\mathrm{NN}}
\end{array}\right],
$$

so in particular

$$
\left\{\begin{array}{l}
U_{\mathrm{NN}}^{\prime}(t)=\frac{\partial \bar{X}^{\mathrm{N}}}{\partial r}\left(\phi_{\bar{X}}(t, p)\right) U_{\mathrm{NN}} \\
U_{\mathrm{NN}}(0)=1
\end{array}\right.
$$

By Property 2 it follows that this last function only depends on $j_{k} X(0)$ since $\forall t \in \mathbb{R}: \phi_{\bar{X}}(t, p) \in S^{2} \times\{0\}$.

\section{Proof of Theorem 1}

We look for a homeomorphism $h$, realizing an equivalence between $\bar{X}$ and $\bar{X}_{k}$, with the following properties. First of all we take $h \mid S^{2} \times\{0\}$ to be the identity, because of Corollary 1. Write $h=\left(h^{\mathrm{T}}, h^{\mathrm{N}}\right)$. We say that $h$ is normally tangent to the identity on a set $A \subset S^{2} \times\{0\}$ if there exist $K>0$ and $\left.\alpha \in\right] 0,1[$ such that for all $u \in A$ and small $r$ we have:

$$
\left|\frac{h^{\mathrm{T}}(u, r)-u}{r^{\alpha}}\right| \leq K \text { and }\left|\frac{h^{\mathrm{N}}(u, r)-r}{r^{1+\alpha}}\right| \leq K .
$$

Step 1. We start the construction of $h$ at the saddle points of $\bar{X} \mid S^{2} \times\{0\}$. Let $q$ be such a saddle. By Corollary 2(a) the eigenvalues of $\bar{X}$ and $\bar{X}_{k}$ at $q$ are equal. Therefore, according to Theorem $A$ in Appendix 1, we can find a neighbourhood $V$ of $q$ in $S^{2} \times \mathbb{R}$ and a homeomorphism $h$ defined on $V$ conjugating $\bar{X} \mid V$ and $\bar{X}_{k} \mid h(V)$ such that $h$ is normally tangent to the identity on $V \cap\left(S^{2} \times\{0\}\right)$. In this way we get for each saddle $q$ of $\bar{X} \mid S^{2} \times\{0\}$ a neighbourhoood $V(q)$ of $q$ and a conjugacy $h: V(q) \rightarrow h(V(q))$ between $\bar{X}$ and $\bar{X}_{k} \mid V$. Choose these neighbourhoods $V(q)$ so small that the saturations $\bigcup_{t \in \mathbf{R}} \phi_{\bar{X}}(t, V(q))$ of $V(q)$ are disjoint for distinct saddles. (And similarly the $\bar{X}_{k}$-orbit saturations of $h(V(q))$ are disjoint.) This is possible since we do not allow saddle connections.

Step 2. Let $p$ be a source of $\bar{X} \mid S^{2} \times\{0\}$. Take a small closed curve $C$ in $S^{2}$ around $p$ such that the orbits of $\bar{X} \mid S^{2} \times\{0\}$ are transversal to $C$. Then for small $\delta>0$ the orbits of $\bar{X}$ are transversal to $C \times]-\delta, \delta$ [. If no stable manifold of a saddle-point of $\bar{X} \mid S^{2} \times\{0\}$ intersects $C$ then we just take $h$ to be the identity on $C \times[-\delta, \delta]$. Otherwise let $q_{1}, \ldots, q_{n}$ be the saddles of $\bar{X} \mid S^{2} \times\{0\}$ such that their stable manifolds intersected with $S^{2} \times\{0\}$ cut C. We denote $\left\{s_{i}\right\}=W^{s}\left(q_{i}\right) \cap\left(S^{2} \times\{0\}\right) \cap C$ (see figure 


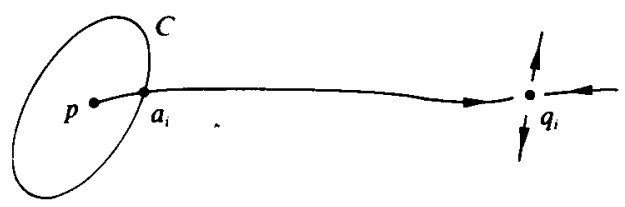

FIGURE 1

1). Now we extend $h$, defined in Step 1, by means of saturation in negative time, until we hit $C \times]-\delta, \delta\left[\right.$. More precisely, for each $i \in\{1, \ldots, n\}$ let $V_{i}$ be a neighbourhood of $q_{i}$ on which $h$ is defined in Step 1 and let $U_{i}=\left\{\phi_{\bar{x}}(t, x) ; x \in V_{i}\right.$ and $t(x) \leq t<0\}$ where $t(x)$ is the first time the negative orbit through $x$ hits $C \times]-\delta, \delta[$, i.e. $\left.\phi_{\bar{X}}(t(x), x) \in C \times\right]-\delta, \delta\left[\right.$; this is well defined if $V_{i}$ is small enough (see figure 2).

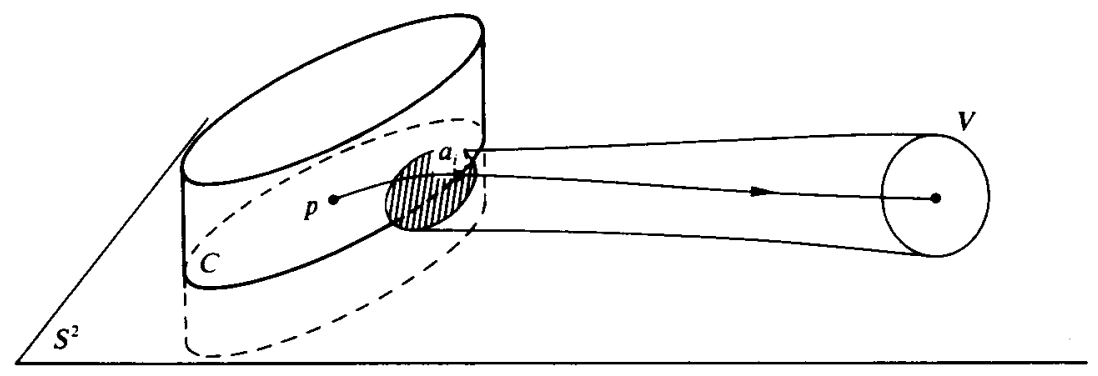

Figure 2

For $y \in U_{i}$ we define $h(y)=\phi_{\bar{X}_{k}}\left(t, h\left(\phi_{\bar{X}}(-t, y)\right)\right)$, that is: we force $h$ to be a conjugacy on $U_{i}$. This implies that $h$ is the identity on $U_{i} \cap\left(S^{2} \times\{0\}\right)$. We check that $h$ is still normally tangent to the identity on $U_{i} \cap(C \times\{0\})$ in the following lemma.

LEMMA. Let $A \subset S^{2} \times\{0\}$ be a line segment or a closed curve transverse to the $\bar{X}$-orbits. Let $t: A \rightarrow \mathbb{R}_{0}^{+}$be a given $C^{\infty}$ function and put $B=\left\{\phi_{\bar{X}}(t(x), x) ; x \in A\right\}$. If $h$ is an equivalence between $\bar{X}$ and $\bar{X}_{k}$ on a neighbourhood $V$ of $\left\{\phi_{\bar{X}}(s, x) ; x \in A\right.$ and $0 \leq s \leq$ $t(x)\}$ in $S^{2} \times \mathbb{R}$, and if $h$ is normally tangent to the identity on $A$, then $h$ is normally tangent to the identity on $B$.

Proof. We take coordinates $\left(u_{1}, u_{2}, r\right)$ in which locally $A \subset\left\{r=u_{2}=0\right\}$ and $B \subset\{r=0$ and $\left.u_{2}=u_{2}^{0}\right\}$ for some $u_{2}^{0}$ and such that the orbits of $\bar{X}$ are 'horizontal' lines $\left\{u_{1}=\right.$ constant and $r=$ constant $\}$. From Property 2 we know that $\bar{X}_{\mathrm{N}}-\bar{X}_{k, \mathrm{~N}}=r^{2} f_{\mathrm{N}}$. We put $f_{0}=\sup \left\{\left|f_{\mathrm{N}}(x)\right| ; x \in V\right\}$, which is finite for small $V$, and $m_{0}=$ inf $\left\{u_{2}\right.$-component of $\left.\bar{X}_{k}(x) ; x \in V\right\}$. For small $V$ we have $m_{0}>0$. Let $\left(u_{1}, 0, r\right) \in V$ be given and put $\bar{r}=h_{\mathrm{N}}\left(u_{1}, 0, r\right)$. We know that $\bar{r}=r\left(1+k\left(u_{1}, 0, r\right)\right)$ with $\left|k\left(u_{1}, 0, r\right)\right| \leq K r^{\alpha}$. Consider the two initial value problems (see figure 3 )

$$
\left\{\begin{array}{l}
\frac{d R_{1}}{d u_{2}}=-\frac{R_{1}^{2} f_{0}}{m_{0}} \\
R_{1}(0)=\bar{r}
\end{array}\right.
$$




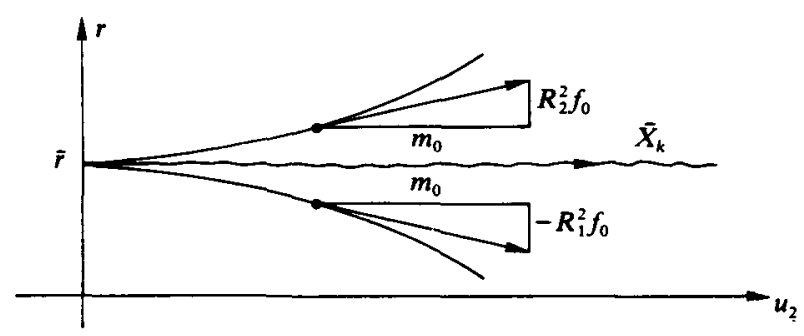

Figure 3. Front view.

and

$$
\left\{\begin{array}{l}
\frac{d R_{2}}{d u_{2}}=\frac{R_{2}^{2} f_{0}}{m_{0}} \\
R_{2}(0)=\bar{r}
\end{array}\right.
$$

We can solve them easily:

$$
R_{1}\left(u_{2}\right)=\frac{\bar{r}}{1+\frac{f_{0}}{m_{0}} u_{2} \bar{r}}
$$

and

$$
R_{2}\left(u_{2}\right)=\frac{\bar{r}}{1-\frac{f_{0}}{m_{0}} u_{2} \bar{r}} .
$$

If $V$ is small enough we can write for all $u_{2} \in\left[0, u_{2}^{0}\right]$ :

$$
R_{2}\left(u_{2}\right) \leq \frac{\bar{r}}{1-\frac{f_{0}}{m_{0}} u_{2}^{0} \bar{r}} \leq \frac{r\left(1+K r^{\alpha}\right)}{1-\frac{f_{0}}{m_{0}} \cdot u_{2}^{0} \cdot 2 r} \leq r\left(1+K_{2} r^{\alpha}\right)
$$

for some $K_{2}>0$. Here we have used $\left.\left.\alpha \in\right] 0,1\right]$. Similarly:

$$
R_{1}\left(u_{2}\right) \geq r\left(1-K_{3} r^{\alpha}\right)
$$

for some $K_{3}>0$. From this and $\bar{X}_{\mathrm{N}}-\bar{X}_{k, \mathrm{~N}}=r^{2} f_{\mathrm{N}}$ it follows that

$$
r\left(1-K_{3} r^{\alpha}\right) \leq h_{N}\left(u_{1}, u_{2}^{0}, r\right) \leq r\left(1+K_{2} r^{\alpha}\right) \text {. }
$$

In the same way, using $\bar{X}_{\mathrm{T}}-\bar{X}_{k, \mathrm{~T}}=r f_{\mathrm{T}}$, we obtain the required estimates for $h_{\mathrm{T}}$. This proves the lemma.

So far $h$ is defined on $U_{i} \cap(C \times \mathbb{R})$. We continue Step 2 by extending $h$ to a neighbourhood of $C \times\{0\}$ in $C \times]-\delta, \delta[$ as follows. We have to take care that $h$ is normally tangential to the identity on $C \times\{0\}$. We may assume that the neighbourhoods $V_{i}$ are chosen such that $U_{i} \cap(C \times]-\delta, \delta[)$ are 'vertical' strips of the form $\left.\left[a_{i}, b_{i}\right] \times\right]-\delta, \delta\left[\right.$, where the $\left[a_{i}, b_{i}\right]$ are disjoint segments in $C$.

We restrict the construction to the 'exterior' region $\{r \geq 0\}$, since the construction for $\{r \leq 0\}$ will be similar and can be pasted decently. We consider first, instead of $h$, the following homeomorphism $\tilde{h}$ : for $\left.x \in\left[a_{i}, b_{i}\right] \times\right]-\delta, \delta[$ we define $\tilde{h}(x)$ to be 
the intersection point of the orbit of $\bar{X}_{k}$ through $h(x)$ with $\left.C \times\right]-\delta, \delta[$. (See figure 4.) $\tilde{h}$ is obtained as the restriction of a new equivalence derived from the conjugacy $h$, hence it follows from the lemma that $\tilde{h}$ is 'tangent to the identity'. Since $\tilde{h}$ maps $U_{i} \cap(C \times]-\delta, \delta[)$ into $C \times \mathbb{R}$ we will first extend $\tilde{h}$ and then use this to find an

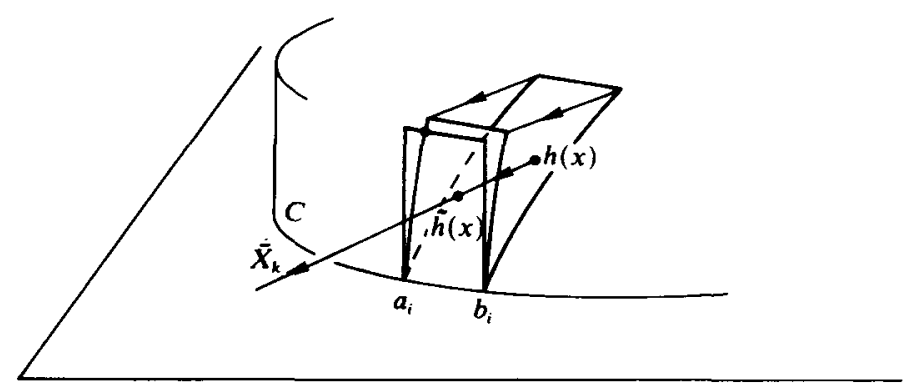

FIGURE 4

extension of $h$. We extend $\tilde{h}$ to a neighbourhood of $C$ in $C \times]-\delta, \delta[$ as follows. Let $i \in\{1, \ldots, n\}$; we denote $a_{n+1}=a_{1}, b_{n+1}=b_{i}$. In between $b_{i}$ and $a_{i+1}$ we fix a line $\left\{c_{i}\right\} \times\left[0, \eta_{i}\right]$, where $0<\eta_{i}<1$ is small and fixed; we define $\tilde{h} \mid\left\{c_{i}\right\} \times\left[0, \eta_{i}\right]$ to be the identity.

Let $r_{j}=j^{-1} \eta_{i}$ for all $j \in \mathbb{N}$. We define $\tilde{h}$, by induction on $j$, on a rectangle $\left[b_{i}, c_{i}\right] \times$ $\left[r_{j+1}, r_{j}\right]$ as follows. We denote

$$
Z(j)=\left\{(u, r) \in C \times\left[0, \delta\left[\mid r_{j+1}-K r_{j+1}^{1+\alpha}<r<r_{j}+K r_{j}^{1+\alpha}\right\}\right.\right.
$$

where $K$ and $\alpha$ are so that

$$
\left|\frac{\tilde{h}_{N}(u, r)}{r}-1\right| \leq K r^{\alpha} \quad \text { for all } u \in \bigcup_{i=1}^{n}\left[a_{i}, b_{i}\right]
$$

and small $r>0$.

Define $\varepsilon(r)=K_{4} r^{\alpha}$, where $K_{4}$ is so that

$$
h\left(\left\{b_{i}\right\} \times\left[0, r_{1}\right]\right) \subset\left\{(u, r)|| u-b_{i} \mid<\varepsilon(r)\right\} .
$$

This is possible since $\tilde{h}$ is normally tangent to the identity at $\left(b_{1}, 0\right)$.

We connect, by induction for all $j, \tilde{h}\left(b_{i}, r_{j}\right)$ to $\left(c_{i}, r_{j}\right)$ by means of an injective $C^{0}$ arc $\tau_{j}:\left\{b_{i}, c_{i}\right] \rightarrow Z(j-1) \cap Z(j)$ in such a way that $\tau_{j}\left(b_{i}\right)=\tilde{h}\left(b_{i}, r_{j}\right)$ and for all $u \in\left[\varepsilon\left(r_{j}\right), c_{i}\right]: \tau_{j}(u)=\left(u, r_{j}\right)$. Also $\left.\left.\tau_{j}(] b_{i}, c_{i}\right]\right)$ does not meet $\operatorname{Im} \tau_{1}, \ldots, \operatorname{Im} \tau_{j-1}$ and $h\left(\left\{b_{i}\right\} \times\left[0, r_{1}\right]\right)$. We define $\tilde{h}$ on $\left[b_{i}, c_{i}\right] \times\left\{r_{j}\right\}$ by $\tilde{h}\left(u, r_{j}\right)=\tau_{j}(u)$ and moreover $\tilde{h}$ is the identity on $\left\{(u, r) \mid u \geq b_{i}+\varepsilon(r)\right\}$. In this way $\tilde{h}$ is defined on the boundary of the rectangle $\left[b_{i}, c_{i}\right] \times\left[r_{j+1}, r_{j}\right]$. The image of this curve is a curve without selfintersections. Now we apply the Schönflies theorem [Mo] to extend $\tilde{h}$ to $\left[b_{i}, c_{i}\right] \times$ $\left[r_{j+1}, r_{j}\right]$ for all $j$. So $\tilde{h}\left(\left[b_{i}, c_{i}\right] \times\left[r_{j+1}, r_{j}\right]\right) \subset Z(j)$.

First of all we note that $\tilde{h}$ extends to the identity on $\left[b_{i}, c_{i}\right] \times\{0\}$. Next we check that $\tilde{h}$ is normally tangent to the identity on $\left[b_{i}, c_{i}\right] \times\{0\}:$ let $r \in\left[r_{j+1}, r_{j}\right]$ and $u \in\left[b_{i}, c_{i}\right]$. We know that

$$
\tilde{h}_{N}(u, r)<r_{j}+K r_{j}^{1+\alpha}
$$


Since $r_{j} / r_{j+1} \leq 2$ for all $j \in \mathbb{N}$, this implies

$$
\begin{aligned}
\frac{\tilde{h}_{N}(u, r)-r}{r} & \leq \frac{r_{j}-r+K r_{j}^{1+\alpha}}{r} \leq \frac{K r_{j}^{1+\alpha}}{r_{j+1}} \\
& \leq K\left(\frac{r_{j}}{r_{j+1}}\right)^{1+\alpha} r^{\alpha} \leq 2^{1+\alpha} K r^{\alpha} .
\end{aligned}
$$

In a similar way we find a constant $K_{5}$ such that

$$
\frac{r-\tilde{h}_{N}(u, r)}{r} \leq K_{5} r^{\alpha}
$$

On the other hand for $(u, r)$ with $\left|u-b_{i}\right| \leq \varepsilon(r)=K_{4} r^{\alpha}$ we know that $(U, R):=\tilde{h}(u, r)$ also satisfies $\left|U-b_{i}\right| \leq K_{4} R^{\alpha}$, by construction, and as $R=r+O\left(r^{1+\alpha}\right)$ we have $|U-u| \leq 2 K_{4} R^{\alpha}$, hence there exists a $K_{6}>0$ such that $|U-u| \leq K_{6} r^{\alpha}$; for $(u, r)$ with $u \in\left[\varepsilon(r), c_{i}\right]$ this is trivially true. So on $\left[b_{i}, c_{i}\right] \times\left[0, \eta_{i}\right]$ :

$$
\left|\frac{\tilde{h}_{T}(u, r)-u}{r^{\alpha}}\right| \leq K_{6} .
$$

By repeating the same construction to the regions $\left[c_{i}, a_{i+1}\right]$ we can assume now that $\tilde{h}$ is defined on, say, $C \times[-\eta, \eta]$.

There exists a continuous function

$$
t: \tilde{h}\left(\bigcup_{i=1}^{n}\left[a_{i}, b_{i}\right] \times\left[0, \eta_{i}\right]\right) \rightarrow \mathbb{R}
$$

such that $\phi_{\bar{X}_{k}}(t(\tilde{h}(x)), \tilde{h}(x))=h(x)$. Let $t^{*}$ denote any continuous extension of $t$ to $C \times[-\eta, \eta]$. Finally put $h(x)=\phi_{\bar{x}_{k}}\left(t^{*}(\tilde{h}(x)), \tilde{h}(x)\right)$ for all $x \in C \times[-\eta, \eta]$. For the same reasons as in the lemma above $h$ is normally tangent to the identity on $C \times\{0\}$. Using Theorem B in Appendix 2 we extend $h$ to a conjugacy on a neighbourhood of the source $p$ of $\bar{X} \mid S^{2} \times\{0\}$.

Step 3. Let $\gamma \subset S^{2} \times\{0\}$ be a repelling limit cycle of $\bar{X} \mid S^{2} \times\{0\}$. Let $\Sigma$ and $\Sigma_{1} \subset \Sigma$ be small transversal sections of $\gamma$ on which the Poincaré maps $P: \Sigma_{1} \rightarrow \Sigma$ for $\bar{X}$ and $P_{k}: \Sigma_{1} \rightarrow \Sigma$ for $\bar{X}_{k}$ are defined. By assumption (2) in the statement of Theorem 1, $P$ and $P_{k}$ are hyperbolic.

$P \mid \Sigma_{1} \cap\left(S^{2} \times\{0\}\right)$ is repelling. We make a similar construction as in Step 2 as follows. Take a smooth closed curve $D$ in $S^{2}$ around $\gamma$ in a small neighbourhood of $\gamma$ such that $\bar{X} \mid S^{2} \times\{0\}$ is transversal to $D$. For small $\delta>0$ we still have transversality to $D \times]-\delta, \delta\left[\right.$. Just as in Step 2 we consider all the saddles of $\vec{X} \mid S^{2} \times\{0\}$ whose stable manifolds, intersected with $S^{2} \times\{0\}$, cut $D$. We extend $h$ obtained in Step 1 in negative time starting at the neighbourhoods of these saddles, until we hit $D \times]-\delta, \delta[$; this is done in the same way as in Step 2 . We continue Step 3 by extending $h$ to a neighbourhood of $D \times\{0\}$ in $D \times \mathbb{R}$. This is done precisely as in Step 2. The obtained extension, always called $h$, is also normally tangent to the identity on $D \times\{0\}$. If no stable manifold of a saddle of $\bar{X} \mid S^{2} \times\{0\}$ intersects $D$ then we just take $h$ to be the identity on a neighbourhood of $D \times\{0\}$ in $D \times \mathbb{R}$. Finally we repeat the whole construction of this Step 3 , only using this time a transversal cylinder of the form $D \times]-\delta, \delta[$ with $D$ on the other side of $\gamma$. 
Step 4. Let $\gamma \subset S^{2} \times\{0\}$ be a repelling limit cycle of $\bar{X} \mid S^{2} \times\{0\}$ such that its Poincaré map is of saddle type. We will extend $h$ such that it has an invariant cylinder around $\gamma$ transversal to $\bar{X}$ and $\bar{X}_{k}$. But we will have to switch from conjugacy to equivalence.

Let $D_{1}$ be a closed curve inside $D$ (see Step 3 ) in $S^{2} \times\{0\}$ transversal to $\bar{X}$, and let $\delta_{1}$ be small such that $\bar{X}$ is transversal to $\left.D_{1} \times\right]-\delta_{1}, \delta_{1}[$. For each $x \in D \times]-\delta, \delta[$ there exists a unique $t(x)<0$ such that $\left.\phi_{\bar{X}}(t(x), x) \in D_{1} \times\right]-\delta_{1}, \delta_{1}[$. We extend $h$ to $A:=\left\{\phi_{\bar{X}}(t, x) \mid x \in D \times\right]-\delta, \delta[$ and $t(x)<t<0\}$ by interpolation using arc-length as follows. For $y=\phi_{\bar{X}}(t, x) \in A$ there exists a unique $\left.x \in D \times\right]-\delta, \delta[$ such that $y$ is in the orbit of $x$ for $\bar{X}$. Let $d_{\bar{X}}$ resp. $d_{\bar{X}_{k}}$ be metrics in the orbits of $\bar{X}$ resp. $\bar{X}_{k}$ defined by arc length. We define $h(y)$ to be the unique element in the negative orbit of $h(x)$ for $\bar{X}_{k}$ such that

$$
d_{\bar{X}_{k}}(h(x), h(y))=\frac{d_{\bar{X}_{k}}\left(h(x), D_{1} \times\right]-\delta_{1}, \delta_{1}[)}{d_{\bar{X}}\left(x, D_{1} \times\right]-\delta_{1}, \delta_{1}[)} d_{\bar{X}}(x, y) .
$$

$h$ is continuous, and by reversing the process we see that it is a homeomorphism; moreover by construction it is an equivalence between $\bar{X}$ and $\bar{X}_{k}$ on $A$.

We conclude that $\left.h\left(D_{1} \times\right]-\delta_{1}, \delta_{1}[) \subset D_{1} \times\right]-\delta_{1}, \delta_{1}[$. We form the same construction on the other side of $\gamma$ in $S^{2} \times\{0\}$. Since $P$ is of saddle type, the normal eigenvalue of $P$ is less than one (it is always positive). Denote $\{a\}=\gamma \cap \Sigma_{1}$. As $h$ is defined on $\left.D_{1} \times\right]-\delta_{1}, \delta_{1}$ [ this automatically induces a conjugacy $H$ between $P$ and $P_{k}$ on the connected component of $\Sigma_{1} \backslash W^{s}(a)$ cut by the orbits of $\left.D_{1} \times\right]-\delta_{1}, \delta_{1}[$ : fix fundamental domains $F$ resp. $F_{k}$ for $P$ resp. $P_{k}$ in this component and take $x \in F$. We let $y$ be the unique point of intersection of the positive orbit of $\bar{X}$ through $x$ with $\left.D_{1} \times\right]-\delta_{1}, \delta_{1}$ [: we let $H(x)$ be the (unique) point of intersection of the negative orbit of $\bar{X}_{k}$ through $h(y)$ with $F_{k}$. By the lemma in Step 2 it follows that $H$ is normally tangential to the identity on $F \cap\left(S^{2} \times\{0\}\right)$.

In the same way $H$ can be defined on the other connected component if $\Sigma_{1} \backslash W^{s}(a)$. Now by Theorem B in Appendix 2, $H$ can be extended to a conjugacy of $P$ and $P_{k}$ on the whole of $\Sigma_{1}$.

Next it is standard to construct from $H$ an equivalence $h$ between $\bar{X}$ and $\bar{X}_{k}$ on a neighbourhood of $\gamma$ in $S^{2} \times \mathbb{R}$, see for example [PM]. We consider once more a transversal cylinder $\left.D_{2} \times\right]-\delta_{2}, \delta_{2}$ [ where $D_{2}$ lies between $\gamma$ and $D_{1}$ (see Step 6); we consider once more a transversal cylinder $\left.D_{3} \times\right]-\delta_{3}, \delta_{3}\left[\right.$ where $D_{3}$ lies between $\gamma$ and $D_{2}$, and we take care that $h$ is defined on $\left.D_{3} \times\right]-\delta_{3}, \delta_{3}$ [ by our construction just made (see figure 5). Precisely as before we can extend $h$ from $\left.D_{3} \times\right]-\delta_{3}, \delta_{3}[$ to $\left.D_{2} \times\right]-\delta_{2}, \delta_{2}\left[\right.$ in such a way that $\left.h\left(D_{2} \times\right]-\delta_{2}, \delta_{2}[) \subset D_{2} \times\right]-\delta_{2}, \delta_{2}[$. The only thing that remains to be done is to make an extension of $h$ to the zone $Z$ between $\left.D_{2} \times\right]-\delta_{2}, \delta_{2}\left[\right.$ and $\left.D_{1} \times\right]-\delta_{1}, \delta_{1}[$. We do this as follows. Remember that $H$ was defined on $\Sigma_{1} \backslash W^{s}(a)$ by means of the definition of $h$ on $\left.D_{1} \times\right]-\delta_{1}, \delta_{1}[$. So let $z \in Z$. Let $y$ be the intersection point of the negative orbit of $\bar{X}$ through $z$ with $D_{2} \times$ ]$-\delta_{2}, \delta_{2}\left[\right.$, and let $y^{\prime}$ be the intersection point of the positive orbit of $\bar{X}$ through $z$ with $\left.D_{1} \times\right]-\delta_{1}, \delta_{1}[$. We define $h(z)$ to be the unique point of the positive orbit of 


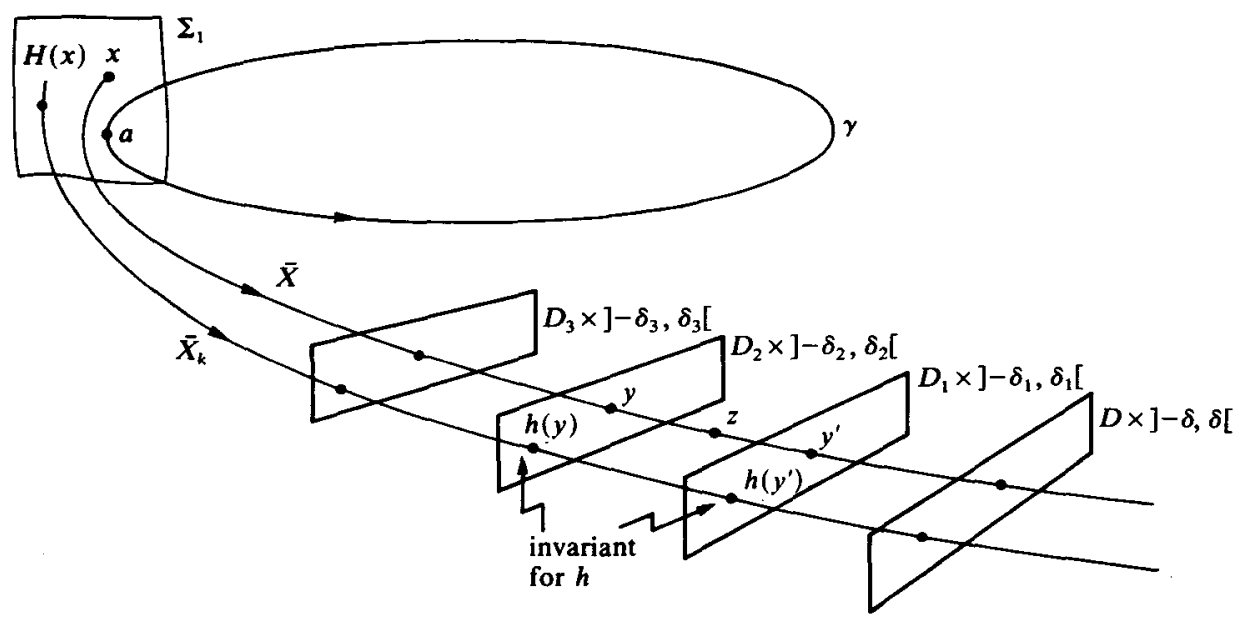

Figure 5

$\bar{X}_{k}$ through $h(y)$ for which

$$
\frac{d_{\bar{X}_{k}}(h(y), h(z))}{d_{\bar{X}_{k}}\left(h(y), h\left(y^{\prime}\right)\right)}=\frac{d_{\bar{X}}(y, z)}{d_{\bar{X}}\left(y, y^{\prime}\right)} .
$$

Finally we make a completely similar construction on the other side of $\gamma$ in $S^{2} \times\{0\}$.

Step 5. Let $\gamma \subset S^{2} \times\{0\}$ be a repellor considered as a periodic orbit of $X$, i.e. the Poincaré-map $P$ is a repellor. Take a narrow solid 2-torus $T$ containing $\gamma$ and transversal to $\bar{X}$. We can suppose that the two transversal cylinders of the form $D \times[-\eta, \eta]$ from Step 3 lie in $\partial T$. Remember that $h$ was defined on these two cylinders (see figure 6 ). Extend $h$ in an arbitrary way to a homeomorphsm on $\partial T$.

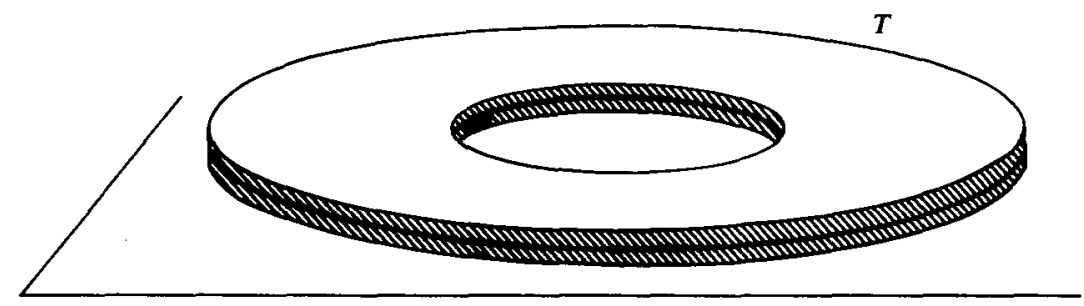

Figure 6

Then extend $h$ to $T \backslash \gamma$ by means of saturation in negative time, that is: for each $x \in T \backslash \gamma$ there exists one $t>0$ and one $y \in \partial T$ such that $\phi_{\bar{X}}(t, x)=y$. We define $h(x)$ to be $\phi_{\bar{X}_{k}}(-t, h(y))$. Remark that $h$ conjugates $\bar{X}$ and $\bar{X}_{k}$ in $T \backslash \gamma$. Indeed, if $\left(x_{i}\right)_{i \in N}$ is a sequence such that $x_{i} \rightarrow \gamma$, then for each $i \in \mathbb{N}$ there exists one $t_{i}>0$ and one $y_{i} \in \partial T$ such that $\phi_{\bar{X}}\left(t_{i}, x_{i}\right)=y_{i}$. Now $t_{i} \rightarrow \infty$, hence for $h\left(x_{i}\right)=\phi_{\bar{X}_{k}}\left(-\partial T t_{i}, h\left(y_{i}\right)\right)$ we obtain that $h\left(x_{i}\right) \rightarrow \gamma$. In order to extend $h$ continuously to a map $h: \gamma \rightarrow \gamma$, we need to reparametrise orbits of $\bar{X}$ is a standard way, see [PM]. 
Step 6. For each sink $r$ or $\bar{X} \mid S^{2} \times\{0\}$ we do the following. Let $E$ be a small closed curve in $S^{2}$ around $r$, transversal to $\bar{X}$. We take a small $\varepsilon>0$ such that the sequel holds. If $x \in E \times[-\varepsilon, \varepsilon]$ then one has three possibilities for the negative orbit of $\bar{X}$ through $x$ : (1) it cuts a neighbourhood of a saddle $q$ of $\bar{X} \mid S^{2} \times\{0\}$ on which $h$ was defined in Step 1; (2) it cuts a set of the form $C \times[-\eta, \eta]$ where $C$ is a small closed curve with center a source $p$ as in Step 2; (3) it cuts a set of the form $D \times[-\eta, \eta]$ near a repelling limit cycle $\gamma$ as in Step 3. Hence we can extend $h$ by means of saturation in positive time until it is defined on $E \times[-\varepsilon, \varepsilon]$. Remark that, up to now, $h$ is a conjugacy, and by the lemma in Step $2, h$ is normally tangential to the identity on $E \times\{0\}$. Again extend $h$ to a neighbourhood of $r$ using Theorem B.

Step 7. Let $\gamma \subset S^{2} \times\{0\}$ be an attracting limit cycle of $\bar{X} \mid S^{2} \times\{0\}$. Let $F$ be smooth closed curve in $S^{2} \times\{0\}$ around $\gamma$ in a small neighbourhood of $\gamma$ such that $\bar{X} \mid S^{2} \times\{0\}$ is transversal to $F$. For small $\varepsilon>0$ we still have transversality to $F \times[-\varepsilon, \varepsilon]$, and just like above we can extend $h$ by means of saturation in positive time until it is defined on $F \times[-\varepsilon, \varepsilon] . h$ is normally tangential to the identity on $F \times\{0\}$ by the lemma in Step 2.

We do the same for a closed transversal curve on the other side of $\gamma$ in $S^{2} \times\{0\}$.

Step 8. We make an exactly analoguous construction as in Step 4 for those attracting limit cycles, sources and sinks of $\bar{X} \mid S^{2} \times\{0\}$ which are, considered in $S^{2} \times \mathbb{R}$, of saddle type. That is: we extend $h$ to smaller transversal cylinders around them such that these cylinders are invariant for $h$.

Step 9. Let $\gamma$ be an attracting limit cycle of $\bar{X} \mid S^{2} \times\{0\}$. In Step 8 we defined $h$ up to transversal invariant cylinders on both sides of $\gamma$. Again from the lemma in Step $2, h$ is normally tangential to the identity, and hence we can proceed precisely as in Steps 4 and 5.

\section{Consequences and remarks}

(1) If we look closely to the proof of Theorem 1 we notice that we only had to switch from conjugacy to equivalence in the neighbourhood of limit cycles. Hence if there are no limit cycles, $\bar{X}$ and $\bar{X}_{k}$ will be $C^{0}$-conjugate. Moreover, as the attracting and the repelling periodic orbits are giving no problem when blowing down $S^{2} \times\{0\}$ to 0 (see Step 8 ), this yields:

THEOREM 2. Let $X$ be as in Theorem 1 and assume that $\vec{X}$ has no periodic orbits of saddle type. Then $X / r^{k-1}$ is $C^{0}$ conjugate to $X_{k} / r^{k-1}$ near 0 .

(2) From the methods of the proof it clearly follows that the condition that $\bar{X} \mid S^{2} \times\{0\}$ is Morse-Smale can be weakened. Let us just mention that the hyperbolicity of the singularities and periodic orbits is not essential. However, it is not permitted that $\bar{X} \mid S^{2} \times\{0\}$ has a saddle-connection between hyperbolic saddles, see [Str].

Appendix 1. A $C^{0}$ normal linearisation theorem providing conjugacies with normal derivative equal to 1

Consider a $C^{2}$ diffeomorphism $f$ on $\mathbb{R}^{n} \times \mathbb{R}$ with a fixed point in $0, f(0)=0$, such that $f\left(\mathbb{R}^{n} \times\{0\}\right)=\mathbb{R}^{n} \times\{0\}$. Take $(y, z) \in \mathbb{R}^{n} \times \mathbb{R}$ and write $f(y, z)=\left(f_{y}(y, z), f_{z}(y, z)\right) \in$ 
$\mathbb{R}^{n} \times \mathbb{R}$. Since $f$ respects $\mathbb{R}^{n} \times\{0\}$, one has

$$
f_{z}(y, z)=\gamma(y, z) \cdot z
$$

with $\gamma(y, z)$ a $C^{1}$-map. Write $a=\gamma(0,0)$.

Theorem A. Suppose $f, f^{\prime}$ are two diffeomorphisms as above with

$$
\begin{aligned}
& f\left|\mathbb{R}^{n} \times\{0\}=f^{\prime}\right| \mathbb{R}^{n} \times\{0\} ; \\
& \gamma(y, 0)=\gamma^{\prime}(y, 0) ;
\end{aligned}
$$

along $\mathbb{R}^{n} \times\{0\}$, (and in particular $\left.a=a^{\prime}\right)$. Finally

$$
|a|\left(=\left|a^{\prime}\right|\right) \neq 1 \text {. }
$$

Then there exists a neighbourhood $U$ of 0 and a homeomorphism $h$ conjugating $f$ and $f^{\prime}$ on $U$, i.e. $h \circ f=f^{\prime} \circ h$, and writing $h=\left(h_{y}, h_{z}\right) \in \mathbb{R}^{n} \times \mathbb{R}$ there exists an $\alpha>0$ such that

$$
\left|\frac{h_{y}(y, z)-y}{z^{\alpha}}\right| \leq 1
$$

and

$$
\left|\frac{h_{z}(y, z)-z}{z^{1+\alpha}}\right| \leq 1 .
$$

Remark. From these estimates it follows that $\partial h_{z} / \partial z$ is well defined along $\mathbb{R}^{n} \times\{0\}$ and identical to 1 .

\section{A.1. Two lemmas in a globalised case}

Before proving Theorem $A$ we will state and prove two lemmas. In $\S 2$ we will reduce Theorem $A$ to these Lemmas.

Consider a linear map $L: \mathbb{R}^{n} \times \mathbb{R} \rightarrow \mathbb{R}^{n} \times \mathbb{R}$ with a matrix of the form

$$
L=\left[\begin{array}{ccc}
L_{-} & 0 & 0 \\
0 & L_{+} & 0 \\
0 & 0 & a
\end{array}\right],
$$

where $L_{-}: \mathbb{R}^{n_{-}} \rightarrow \mathbb{R}^{n_{-}}, L_{+}: \mathbb{R}^{n_{+}} \rightarrow \mathbb{R}^{n_{+}}, a \in \mathbb{R}, \mathbb{R}^{n_{-}} \oplus \mathbb{R}^{n_{+}}=\mathbb{R}^{n}$. For definiteness assume that

$$
a>1 \text {. }
$$

We do not assume that $L_{-}$or $L_{+}$is hyperbolic, or that $L_{-}$is contracting. However we assume that, (writing $\|\cdot\|$ for the operator norm)

$$
\left\{\begin{array}{l}
\left\|L_{-}\right\|<a^{1-\delta_{0}}, \\
\left\|L_{+}^{-1}\right\| \cdot a^{1-\delta_{0}}<1 ;
\end{array}\right.
$$

for some $\left.\delta_{0} \in\right] 0, \frac{1}{2}\left[\right.$. Choose and fix $\left.\delta_{0} \in\right] 0, \frac{1}{2}[$ such that (A1.2) holds.

For $\varepsilon, C>0$ consider the class $A(\varepsilon, C)$ of $C^{2}$-maps $\lambda: \mathbb{R}^{n} \times \mathbb{R} \rightarrow \mathbb{R}^{n} \times \mathbb{R}$ such that

$$
\begin{gathered}
\operatorname{supp}(\lambda) \subseteq \mathbb{R}^{n} \times[-\varepsilon, \varepsilon], \quad \operatorname{supp}(\lambda) \text { is compact; } \\
|\lambda| \leq C \cdot \varepsilon, \quad \operatorname{Lip}(\lambda) \leq C, \\
\left|\frac{\lambda(y, z)-\lambda\left(y^{\prime}, z\right)}{y-y^{\prime}}\right| \leq C \cdot \varepsilon ;
\end{gathered}
$$


write $\lambda=\left(\lambda_{-}, \lambda_{+}, \lambda_{z}\right) \in \mathbb{R}^{n_{-}} \times \mathbb{R}^{n_{+}} \times \mathbb{R}$, then one has

$\lambda_{2}(y, 0)=0, \forall y \in \mathbb{R}^{n}=\mathbb{R}^{n}-\times \mathbb{R}^{n^{+}}$i.e. we can write

$\lambda_{z}(y, z)=\gamma(y, z) \cdot z$, with $\gamma C^{1}$;

$$
|\gamma| \leq C \cdot \varepsilon, \quad \operatorname{Lip}(\gamma) \leq C .
$$

Remark. Condition (A1.3)(c) implies that $f=L+\lambda$ satisfies $f\left(\mathbb{R}^{n} \times\{0\}\right) \subset \mathbb{R}^{n} \times\{0\}$.

Now take two diffeomorphisms $f$ and $f^{\prime}$ of the form $f=L+\lambda, f^{\prime}=L+\lambda^{\prime}$ where $L$ is of the form as above, and $\lambda, \lambda^{\prime} \in A(\varepsilon, C)$. Also assume

$$
f\left|\mathbb{R}^{n} \times\{0\}=f^{\prime}\right| \mathbb{R}^{n} \times\{0\} .
$$

Clearly this implies that

$$
\lambda=\lambda^{\prime} \quad \text { along } \mathbb{R}^{n} \times\{0\} .
$$

LemmA A.1. Let $L, C, a, \delta_{0}$ be as above. For any $\tau>a$ and $\delta$ sufficiently close to $\delta_{0}$ there exists a small $\varepsilon>0$ with the following property. Take two diffeomorphisms $f, f^{\prime}$ of the form $f=L+\lambda, f^{\prime}=L+\lambda^{\prime}$, with

$$
\begin{gathered}
\lambda, \lambda^{\prime} \in A(\varepsilon, C) \\
f\left|\mathbb{R}^{n} \times\{0\}=f^{\prime}\right| \mathbb{R}^{n} \times\{0\}
\end{gathered}
$$

the functions $\gamma$ and $\gamma^{\prime}$ from (A1.3)(c) satisfy:

$$
\gamma(y, 0)=\gamma^{\prime}(y, 0), \forall y \in \mathbb{R}^{n} .
$$

Then there exists a unique mapping $h$ such that $h \circ f=f^{\prime} \circ h$, of the form $h=\mathrm{id}+g$, with $\mathbf{g}=\left(\mathrm{g}_{-}, \mathrm{g}_{+}, \mathrm{g}_{\mathrm{z}}\right): \mathbb{R}^{n_{-}} \times \mathbb{R}^{n_{+}} \times \mathbb{R} \rightarrow \mathbb{R}^{n_{-}} \times \mathbb{R}^{n_{+}} \times \mathbb{R}$ satisfying

$$
\begin{aligned}
& \quad g \text { is uniformly continuous on } \mathbb{R}^{n} \times[-\tau \varepsilon, \tau \varepsilon], \\
& \quad g_{+}=0 \text { outside }|z| \leq \tau \cdot \varepsilon, \\
& \left.\mid \begin{array}{l}
\frac{g_{ \pm}(y, z)}{z^{1-\delta}} \mid \leq 1 ; \\
\left|\frac{g_{z}(y, z)}{z^{2-2 \delta}}\right| \leq 1,
\end{array}\right\} \text { for all }(y, z) \in \mathbb{R}^{n} \times[-\tau \varepsilon, \tau \varepsilon] .
\end{aligned}
$$

\section{Remarks}

(1) Property (A1.7)(d) implies that $\left|h_{z}(y, z) / z\right| \rightarrow 1$ uniformly as $z \rightarrow 0$, and therefore in particular that $(\partial / \partial z) h_{z}$ exists along $\mathbb{R}^{n} \times\{0\}$.

(2) We shall prove Lemma A.1 using a method quite similar to the technique employed by Pugh [Pu] in his proof of Hartman's result.

Proof of Lemma A.1. Fix $\tau>a$ and take $\delta$ (close to $\delta_{0}$ ) such that $\left\|L_{-}\right\|<a^{1-\delta}$ and $\left\|L_{+}^{-1}\right\| a^{1-\delta}<1$. Denote for a function $u: \mathbb{R}^{n} \times[-\tau \varepsilon, \tau \varepsilon] \rightarrow \mathbb{R}^{k}$ the supremum norm of $u$ by $|u|$. In the following we shall repeatedly write $g$ in the decomposed form $g=\left(g_{-}, g_{+}, g_{z}\right) \in \mathbb{R}^{n} \times \mathbb{R}^{n} \times \mathbb{R}$. Also we shall use $y$ for points in $\mathbb{R}^{n}$ and $z$ for points in $\mathbb{R}$ giving $(y, z) \in \mathbb{R}^{n} \times \mathbb{R}$. The identity map is denoted as 1 and $1_{z}$ is the map $(y, z) \rightarrow z$.

From now on we shall look at maps

$$
g=\left(g_{-}, g_{+}, g_{z}\right): \mathbb{R}^{n_{-}} \times \mathbb{R}^{n_{+}} \times \mathbb{R} \rightarrow \mathbb{R}^{n_{+}} \times \mathbb{R}^{n_{-}} \times \mathbb{R}
$$


such that the following seminorm is finite:

$$
\|g\|_{\varepsilon}=\sup _{\substack{y \in \mathbb{R}^{n} \\|z| \leq \tau^{\prime} \varepsilon}}\left\{\left|\frac{g_{ \pm}(y, z)}{z^{1-\delta}}\right|,\left|\frac{g_{z}(y, z)}{z^{2-2 \delta}}\right|\right\} .
$$

In fact define the following space $E_{\varepsilon}$

$$
E_{\varepsilon}=\left\{g: \mathbb{R}^{n_{-}} \times \mathbb{R}^{n^{+}} \times \mathbb{R} \rightarrow \mathbb{R}^{n} \times \mathbb{R} ;\right.
$$

$g$ is uniformly continuous, $g_{+}=0$ for $|z| \geq \tau \varepsilon$

and $\left.\|g\|_{\varepsilon}<\infty\right\}$.

Then define $E_{\varepsilon}(r)$ as the $r$-ball in $E_{\varepsilon}$ :

$$
E_{\varepsilon}(r)=\left\{g \in E_{\varepsilon} \mid\|g\|_{\varepsilon} \leqq r\right\} .
$$

We shall show that there exists a conjugacy $h$, between $f$ and $f^{\prime}$, i.e.

$$
h \circ f=f^{\prime} \circ h \text {, }
$$

of the form $h=\mathrm{id}+g, g \in E_{\varepsilon}(1)$. So we need to solve

$$
(1+g) \circ(L+\lambda)=\left(L+\lambda^{\prime}\right) \circ(1+g)
$$

or

$$
g \circ f=L g+\lambda^{\prime} \circ(1+g)-\lambda
$$

This is equivalent to

$$
g=\left[L g+\lambda^{\prime}(1+g)-\lambda\right] \circ f^{-1} .
$$

So expanding (A1.8) in the decomposition $g=\left(g_{-}, g_{+}, g_{z}\right) \in \mathbb{R}^{n_{-}} \times \mathbb{R}^{n_{+}} \times \mathbb{R}$ one gets

$$
\begin{aligned}
& g_{-}=\left[L_{-} g_{-}+\lambda_{-}^{\prime} \circ(1+g)-\lambda_{-}\right] \circ f^{-1}, \\
& g_{+}=\left[L_{+} g_{+}+\lambda_{+}^{\prime} \circ(1+g)-\lambda_{+}\right] \circ f^{-1}, \\
& g_{z}=\left[a \cdot g_{z}+\lambda_{z}^{\prime} \circ(1+g)-\lambda_{z}\right] \circ f^{-1} .
\end{aligned}
$$

Equation (A1.9)(b) is equivalent to

$$
g_{+}=L_{+}^{-1}\left[g_{+} \circ f+\lambda_{+}-\lambda_{+}^{\prime} \circ(1+g)\right]
$$

Now define a new map $P g: \mathbb{R}^{n} \times \mathbb{R} \rightarrow \mathbb{R}^{n-} \times \mathbb{R}^{n+} \times \mathbb{R}$, for maps $g \in E_{\varepsilon}$, by $P g=\left((P g)_{-}\right.$, $\left.(P g)_{+},(P g)_{z}\right)$, where

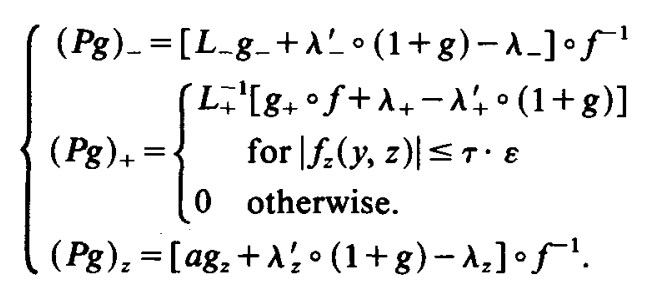

As $\left(E_{\varepsilon},\|\cdot\|_{\varepsilon}\right)$ is a semi-normed space we pass to the quotient space $\tilde{E}_{\varepsilon}$ with respect to the equivalence relation $\sim$ defined by: $g_{1} \sim g_{2} \Leftrightarrow\left\|g_{1}-g_{2}\right\|_{\varepsilon}=0$. On $\tilde{E}_{\varepsilon}$ we keep the notation $\|\cdot\|_{\varepsilon}$. Observe that $\left(\tilde{E}_{\varepsilon},\|\cdot\|_{\varepsilon}\right)$ is sequentially complete: take a Cauchy sequence of classes $\left(\tilde{\mathbf{g}}_{n}\right)_{n \in N}$ in $\left(\tilde{E}_{\varepsilon},\|\cdot\|_{\varepsilon}\right)$; then $g_{n} \mid \mathbb{R}^{n} \times[-\tau \varepsilon, \tau \varepsilon]$ converges uniformly on $\mathbb{R}^{n} \times[-\tau \varepsilon, \tau \varepsilon]$ to some uniformly continuous map $g: g: \mathbb{R}^{n} \times[-\tau \varepsilon, \tau \varepsilon] \rightarrow \mathbb{R}^{n} \times \mathbb{R}$; take any uniformly continuous extension, still called $g: \mathbb{R}^{n} \times \mathbb{R} \rightarrow \mathbb{R}^{n} \times \mathbb{R}$. Then $g \in E_{\varepsilon}$ and $\left\|\tilde{\boldsymbol{g}}_{n}-\tilde{\boldsymbol{g}}\right\|_{\varepsilon} \rightarrow 0$ as $n \rightarrow \infty$. 
Proposition A1.1.

and

$$
\left|\frac{\left(f^{-1}(y, z)\right)_{z}}{z}\right| \in\left[(a+\varepsilon)^{-1},(a-\varepsilon)^{-1}\right]
$$

$$
\left|\frac{(f(y, z))_{z}}{z}\right| \in[a-\varepsilon, a+\varepsilon] .
$$

Proof. This follows immediately from $f=L+\lambda$ and $\left|\lambda_{z}\right| \leq \varepsilon .|z|$.

Proposition A1.2. There exists an $\varepsilon>0$ such that for all $g \in E_{\varepsilon}(1): P g$ is uniformly continuous.

Proof. We have to show that $(P g)_{+}(y, z)$ is continuous. So we have to show that $(P g)_{+}(y, z)=0$ for $\left|f_{z}(y, z)\right|=\tau \varepsilon$. So take $\left|f_{z}(y, z)\right|=\tau \varepsilon$. Since $g_{+}(y, z) \equiv 0$ for $|z| \geq$ $\tau \cdot \varepsilon$, we have $L_{+}^{-1}\left[g_{+} \circ f\right](y, z)=0$. Furthermore from Proposition A1.1, $\left|f_{z}(y, z)\right|=\tau \varepsilon$ implies

$$
\varepsilon \leq \tau \varepsilon \cdot(a+\varepsilon)^{-1} \leq|z| \leq \tau \varepsilon \cdot(a-\varepsilon)^{-1} \leq \tau \varepsilon,
$$

for $\varepsilon>0$ sufficiently small. Since $g \in E_{\varepsilon}(1)$, one has $\left|g_{z}\right| \leq|z|^{2-2 \delta}$ for $|z| \leq \tau \cdot \varepsilon$. Therefore, since $\delta \in] 0, \frac{1}{2}[$, for $|z| \leq \tau \cdot \varepsilon$

$$
\begin{aligned}
\left|(1+g)_{z}\right| & \geq|z|-|z|^{2-2 \delta} \\
& \geq(a+\varepsilon)^{-1} \cdot \tau \varepsilon-(\tau \varepsilon)^{2-2 \delta} \geq \varepsilon,
\end{aligned}
$$

provided $\varepsilon>0$ is sufficiently small. Since $\operatorname{supp}(\lambda) \subseteq \mathbb{R}^{n} \times[-\varepsilon, \varepsilon]$, this implies that $\lambda_{+}(y, z)=0$ and $\lambda_{+}^{\prime}(1+g)=0$ if $\left|f_{z}(y, z)\right|=\tau \cdot \varepsilon$. This implies the continuity of $\mathrm{Pg}$. The uniform continuity of $\mathrm{Pg}$ follows from the fact that $\lambda$ has a compact support.

Remark. In fact if $|z| \geq(a-\varepsilon)^{-1} \cdot \tau \cdot \varepsilon$, then $\left|f_{z}(y, z)\right| \geq \tau \varepsilon$ and $(P g)_{+}(y, z)=0$.

Proposition A1.3. $P$ maps $E_{\varepsilon}(1)$ into $E_{\varepsilon}(1)$ provided $\varepsilon>0$ is sufficiently small.

Proof.(a) First we estimate $(\mathrm{Pg})_{+}$:

using Proposition A1.1

$$
\begin{aligned}
\left|\frac{(P g)_{+}}{z^{1-\delta}}\right| & \leq\left\|L_{+}^{-1}\right\| \cdot\left\{\left|\frac{g_{+} \circ f}{z^{1-\delta}}\right|+\left|\frac{\lambda_{+}-\lambda_{+}^{\prime}(1+g)}{z^{1-\delta}}\right|\right\} \\
& =\left\|L_{+}^{-1}\right\| \cdot\left\{\left|\frac{g_{+}^{\circ} f}{f_{z}^{1-\delta}}\right| \cdot\left|\frac{f_{z}}{z}\right|^{1-\delta}+\left|\frac{\lambda_{+}-\lambda_{+}^{\prime}(1+g)}{z^{1-\delta}}\right|\right\}
\end{aligned}
$$

$$
\leq\left\|L_{+}^{-1}\right\| \cdot\left\{(a+\varepsilon)^{1-\delta} \cdot\left|\frac{g_{+}}{z^{1-\delta}}\right|+\left|\frac{\lambda_{+}-\lambda_{+}^{\prime}(1+g)}{z^{1-\delta}}\right|\right\} .
$$

Let us estimate the last term. Remark that from $f\left|\mathbb{R}^{n} \times\{0\}=f^{\prime}\right| \mathbb{R}^{n} \times\{0\}$, one has $\lambda(y, 0) \equiv \lambda^{\prime}(y, 0)$. Hence

$$
\begin{aligned}
\left|\frac{\lambda_{+}-\lambda_{+}^{\prime}(1+g)}{z^{1-\delta}}\right| \leq & \left|\frac{\lambda_{+}(y, z)-\lambda_{+}(y, 0)}{z^{1-\delta}}\right|+\left|\frac{\lambda_{+}^{\prime}(y, 0)-\lambda_{+}^{\prime}\left(y+g_{y}, z+g_{y}\right)}{z^{1-\delta}}\right| \\
\leq & \left|\frac{\lambda_{+}(y, z)-\lambda_{+}(y, 0)}{z^{1-\delta}}\right|+\left|\frac{\lambda_{+}^{\prime}(y, 0)-\lambda_{+}^{\prime}\left(y+g_{y}, 0\right)}{z^{1-\delta}}\right| \\
& +\left|\frac{\lambda_{+}^{\prime}\left(y+g_{y}, 0\right)-\lambda_{+}^{\prime}\left(y+g_{y}, z+g_{z}\right)}{z^{1-\delta}}\right| .
\end{aligned}
$$


Using (A1.3)(b) this gives:

$$
\leq C \cdot|z|^{\delta}+C \cdot \varepsilon \cdot\left|\frac{g_{y}}{z^{1-\delta}}\right|+C \cdot\left|\frac{z+g_{z}}{z^{1-\delta}}\right|
$$

and since $|z| \leq \tau \cdot \varepsilon$, and $g \in E_{\varepsilon}(1)$, this implies that the above expression is $o(1)$, where $o(1)$ is a function which only depends on $C$ and $\tau$, and $o(1) \rightarrow 0$ as $\varepsilon \downarrow 0$.

Combining this with the estimate for $(P g)_{+} / z^{1-\delta}$ we already had, one gets

$$
\left|\frac{(P g)_{+}}{z^{1-\delta}}\right| \leq\left\|L_{+}^{-1}\right\| \cdot\left\{(a+\varepsilon)^{1-\delta} \cdot 1+o(1)\right\} \leq 1,
$$

for $\varepsilon>0$ sufficiently small and $\delta$ like in (A1.2).

(b) Now we estimate $(\mathrm{Pg})_{-}$.

$$
\left|\frac{P g_{-}}{z^{1-\delta}}\right| \leq\left\|L_{-}\right\| \cdot\left|\frac{g_{-} \circ f^{-1}(y, z)}{z^{1-\delta}}\right|+\left|\frac{\left(\lambda_{-}^{\prime}(1+g)-\lambda_{-}\right) \circ f^{-1}(y, z)}{z^{1-\delta}}\right| .
$$

As before using $\left|\left(f^{-1}(y, z)\right)_{z} / z\right| \leq(a-\varepsilon)^{-1}$, see Proposition A1.1, one gets:

$$
\leq(a-\varepsilon)^{-1+\delta}\left\{\left\|L_{-}\right\|\left|\frac{g_{-}}{z^{1-\delta}}\right|+\left|\frac{\lambda_{-}^{\prime}(1+g)-\lambda_{-}^{\prime}}{z^{1-\delta}}\right|\right\}
$$

and as before, for $g \in E_{\varepsilon}(1)$,

$$
\leq(a-\varepsilon)^{-1+\delta}\left\{\left\|L_{-}\right\| \cdot 1+o(1)\right\} \leq 1,
$$

for $\varepsilon>0$ sufficiently small and $\delta$ as in (A1.2).

(c) Finally we estimate $(P g)_{z}$.

$$
\left|\frac{(P g)_{z}}{z^{2-2 \delta}}\right| \leq(a+\varepsilon)\left|\frac{g_{z} \circ f^{-1}(y, z)}{z^{2-2 \delta}}\right|+\left|\frac{\left(\lambda_{z}^{\prime}(1+g)-\lambda_{z}\right) \circ f^{-1}(y, z)}{z^{2-2 \delta}}\right|,
$$

and as in (b) using Proposition A1.1,

$$
\leq(a-\varepsilon)^{-2+2 \delta}\left\{(a+\varepsilon)\left|\frac{g_{z}}{z^{2-2 \delta}}\right|+\left|\frac{\lambda_{z}^{\prime}(1+g)-\lambda_{z}}{z^{2-2 \delta}}\right|\right\} .
$$

Let us estimate the last term in this sum. Observe that $\lambda_{z}(y, z)=\gamma(y, z) \cdot z$ and $\gamma(y, 0)=\gamma^{\prime}(y, 0)$. So

$$
\begin{aligned}
\left|\frac{\lambda_{z}^{\prime}\left(y+g_{y}, z+g_{y}\right)-\lambda_{z}(y, z)}{z^{2-2 \delta}}\right|= & \left|\frac{\gamma^{\prime}\left(y+g_{y}, z+g_{z}\right) \cdot\left(z+g_{z}\right)-\gamma(y, z) \cdot z}{z^{2-2 \delta}}\right| \\
\leq & \left|\frac{\left(\gamma^{\prime}\left(y+g_{y}, z+g_{z}\right)-\gamma^{\prime}(y, z)\right) \cdot\left(z+g_{z}\right)}{z^{2-2 \delta}}\right| \\
& +\left|\frac{\gamma^{\prime}(y, z) \cdot\left(z+g_{z}-z\right)}{z^{2-2 \delta}}\right|+\left|\left(\frac{\gamma^{\prime}(y, z)-\gamma^{\prime}(y, 0)}{z^{2-2 \delta}}\right) \cdot z\right| \\
& +\left|\frac{(\gamma(y, 0)-\gamma(y, z)) \cdot z}{z^{2-2 \delta}}\right| \\
\leq & C \cdot\left|\frac{g}{z^{1-\delta}}\right| \cdot|z|^{\delta} \cdot\left(1+\left|\frac{g_{z}}{z}\right|\right) \\
& +C \cdot \varepsilon \cdot\left|\frac{g_{z}}{z^{2-2 \delta}}\right|+C \cdot|z|^{2 \delta}+C \cdot|z|^{2 \delta} \\
\leq & C \cdot|z|^{\delta}(1+1)+C \cdot \varepsilon+2 \cdot C \cdot|z|^{2 \delta} \leq o(1),
\end{aligned}
$$


for all $g \in E_{\varepsilon}(1)$, provided $\varepsilon>0$ is small enough. Combining this with the previous estimate for $\left|(P g)_{z} / z^{2-2 \delta}\right|$ one gets:

$$
\begin{aligned}
\left|\frac{(P g)_{z}}{z^{2-2 \delta}}\right| & \leq(a-\varepsilon)^{-2+2 \delta}\{(a+\varepsilon) \cdot 1+o(1)\} \\
& \leq 1
\end{aligned}
$$

for $\varepsilon>0$ sufficiently small. This finishes the proof.

Proposition A1.4. For $\varepsilon>0$ sufficiently small, $P: E_{\varepsilon}(1) \rightarrow E_{\varepsilon}(1)$ is a contraction.

Proof. Again we estimate each term separately

(a)

$$
\begin{aligned}
\left|\frac{\left(P g-P g^{\prime}\right)_{+}}{z^{1-\delta}}\right| & \\
\leq & \left\|L_{+}^{-1}\right\| \cdot\left\{\left|\frac{\left(g_{+}-g_{+}^{\prime}\right) \circ f}{z^{1-\delta}}\right|+\left|\frac{\lambda_{+}^{\prime}(1+g)-\lambda_{+}^{\prime}\left(1+g^{\prime}\right)}{z^{1-\delta}}\right|\right\} \\
\leq & \left\|L_{+}^{-1}\right\| \cdot\left\{(a+\varepsilon)^{1-\delta} \cdot\left|\frac{g_{+}-g_{+}^{\prime}}{z^{1-\delta}}\right|\right. \\
& +\left|\frac{\lambda_{+}^{\prime}\left(y+g_{y}, z+g_{z}\right)-\lambda_{+}^{\prime}\left(y+g_{y}, z+g_{z}^{\prime}\right)}{z^{1-\delta}}\right| \\
& \left.+\left|\frac{\lambda_{+}^{\prime}\left(y+g_{y}, z+g_{z}^{\prime}\right)-\lambda_{+}^{\prime}\left(y+g_{y}^{\prime}, z+g_{z}^{\prime}\right)}{z^{1-\delta}}\right|\right\} \\
\leq & \left\|L_{+}^{-1}\right\| \cdot\left\{(a+\varepsilon)^{1-\delta} \cdot\left\|g-g^{\prime}\right\|\right. \\
& \left.+C \cdot\left|\frac{g_{z}-g_{z}^{\prime} \mid}{z^{2-2 \delta}}\right| \cdot|z|^{1-\delta}+C \cdot \varepsilon \cdot\left|\frac{g_{y}-g_{y}^{\prime}}{z^{1-\delta}}\right|\right\} \\
\leq & \left\|L_{+}^{-1}\right\| \cdot\left\{(a+\varepsilon)^{1-\delta}+C \cdot(\tau \varepsilon)^{1-\delta}+C \cdot \varepsilon\right\} \cdot\left\|g-g^{\prime}\right\| .
\end{aligned}
$$

Take $0<k<1$, such that $\left\|L_{+}^{-1}\right\| \cdot a^{1-\delta}<k<1$ (see Assumption A1.2). Then for $\varepsilon>0$ sufficiently small,

$$
\leq k \cdot\left\|g-g^{\prime}\right\| \text {. }
$$

(b) Similarly

$$
\begin{aligned}
\left|\frac{\left(P g-P g^{\prime}\right)_{-}}{z^{1-\delta}}\right| \leq & \left\|L_{-}\right\| \cdot\left|\frac{g_{-}^{\circ} f^{-1}-g_{-}^{\prime} \circ f^{-1}}{z^{1-\delta}}\right| \\
& +\left|\frac{\left(\lambda_{-}^{\prime}(1+g)-\lambda_{-}^{\prime}(1+g)\right) \circ f^{-1}}{z^{1-\delta}}\right| \\
\leq & \left\|L_{-}\right\| \cdot \frac{1}{(a-\varepsilon)^{1-\delta}}\left|\frac{g_{-}-g_{-}^{\prime}}{z^{1-\delta}}\right| \\
& +\frac{1}{(a-\varepsilon)^{1-\delta}}\left|\frac{\lambda_{-}^{\prime}(1+g)-\lambda_{-}^{\prime}\left(1+g^{\prime}\right)}{z^{1-\delta}}\right| .
\end{aligned}
$$

As in case (a) one estimates the last term, and gets

$$
\leq \frac{1}{(a-\varepsilon)^{1-\delta}} \cdot\left\{\left\|L_{-}\right\| \cdot\left\|g_{-}-g_{-}^{\prime}\right\|+o(1) \cdot\left\|g_{-}-g_{-}^{\prime}\right\|\right\} .
$$


Taking $0<k<1$, with $\left\|L_{-}\right\| / a^{1-\delta}<k<1$, one gets

$$
\leq k \cdot\left\|g-g^{\prime}\right\|,
$$

provided $\varepsilon>0$ is small enough. Finally

(c) $\left|\frac{\left(P g-P g^{\prime}\right)_{z}}{z^{2-2 \delta}}\right| \leq(a+\varepsilon)\left|\frac{\left(g_{z}-g_{z}^{\prime}\right) \circ f^{-1}}{z^{2-2 \delta}}\right|$

$$
\begin{aligned}
& +\left|\frac{\left(\lambda_{z}^{\prime}(1+g)-\lambda_{z}^{\prime}\left(1+g^{\prime}\right)\right) \circ f^{-1}}{z^{2-2 \delta}}\right| \\
\leq & \frac{1}{(a-\varepsilon)^{2-2 \delta}}\left\{(a+\varepsilon) \cdot\left\|g-g^{\prime}\right\|+\left|\frac{\lambda_{z}^{\prime}(1+g)-\lambda_{z}^{\prime}\left(1+g^{\prime}\right)}{z^{2-2 \delta}}\right|\right\} .
\end{aligned}
$$

Let us estimate the last term in this sum. Remark that $\lambda_{z}(y, z)=\gamma(y, z) \cdot z$, and $\gamma(y, 0)=\gamma^{\prime}(y, 0)$.

$$
\begin{aligned}
& \left|\frac{\lambda_{z}^{\prime}\left(y+g_{y}, z+g_{z}\right)-\lambda_{z}^{\prime}\left(y+g_{y}^{\prime}, z+g_{z}^{\prime}\right)}{z^{2-2 \delta}}\right| \\
& \leq\left|\frac{\gamma^{\prime}\left(y+g_{y}, z+g_{z}\right) \cdot\left(z+g_{z}\right)-\gamma^{\prime}\left(y+g_{y}^{\prime}, z+g_{z}^{\prime}\right) \cdot\left(z+g_{z}^{\prime}\right)}{z^{2-2 \delta}}\right| \\
& \leq\left|\frac{\left(\gamma^{\prime}\left(y+g_{y}, z+g_{z}\right)-\gamma^{\prime}\left(y+g_{y}^{\prime}, z+g_{z}^{\prime}\right)\right) \cdot\left(z+g_{z}\right)}{z^{2-2 \delta}}\right| \\
& \quad+\left|\frac{\gamma^{\prime}\left(y+g_{y}^{\prime}, z+g_{z}^{\prime}\right) \cdot\left(\left(z+g_{z}\right)-\left(z+g_{z}^{\prime}\right)\right)}{z^{2-2 \delta}}\right| \\
& \leq C \cdot\left|\frac{g-g^{\prime}}{z^{1-\delta}}\right| \cdot|z|^{\delta} \cdot\left(1+\left|\frac{g_{z}}{z}\right|\right)+C \cdot \varepsilon\left|\frac{g_{z}-g_{z}^{\prime}}{z^{2-2 \delta}}\right| \\
& \leq o(1) \cdot\left\|g-g^{\prime}\right\| .
\end{aligned}
$$

Putting all this together and taking $0<k<1$, such that $a^{-1}<k<1$, one has

$$
\begin{aligned}
& \leq \frac{1}{(a-\varepsilon)^{2-2 \delta}}\{(a+\varepsilon)+o(1)\} \cdot\left\|g-g^{\prime}\right\| \\
& \leq k \cdot\left\|g-g^{\prime}\right\|,
\end{aligned}
$$

provided $\varepsilon>0$ is sufficiently small. This proves the Proposition.

Proposition A1.5. For $\varepsilon>0$ sufficiently small we have:

(i) for all $g_{1}, g_{2} \in E_{\varepsilon}(1)$ with $g_{1} \sim g_{2}: P g_{1} \sim P g_{2}$ in $E_{\varepsilon}(1)$, so this means that $P$ acts on classes in $\tilde{E}_{\varepsilon}(1)$;

(ii) Define $\tilde{\mathrm{P}}: \tilde{\mathrm{E}}_{\varepsilon}(1) \rightarrow \tilde{\mathrm{E}}_{\varepsilon}(1): \tilde{\mathrm{g}} \rightarrow \widetilde{\mathrm{Pg}}$ (this is well defined by (i)), then $\tilde{\mathrm{P}}$ is a contraction on $\tilde{E}_{\varepsilon}(1)$.

Proof. (i) $g_{1} \sim g_{2}$ means: $\forall(y, z) \in \mathbb{R}^{n} \times[-\tau \varepsilon, \tau \varepsilon]: g_{1}(y, z)=g_{2}(y, z)$. Now let $(y, z) \in$ $\mathbb{R}^{n} \times[-\tau \varepsilon, \tau \varepsilon]$. Then, by Proposition A1.1, $f^{-1}(y, z) \in \mathbb{R}^{n} \times[-\tau \varepsilon, \tau \varepsilon]$, so from the definition of $P$ it immediately follows that $\left(P g_{1}\right)_{-}(y, z)=\left(P g_{2}\right)_{-}(y, z)$ and $\left(P g_{1}\right)_{z}(y, z)=\left(P g_{2}\right)_{z}(y, z)$. Concerning the + direction, we distinguish two cases.

Case 1. $\left|f_{z}(y, z)\right| \leq \tau \varepsilon$. Then this fact together with $|z| \leq \tau \varepsilon$ implies $\left(P g_{1}\right)_{+}(y, z)=$ $\left(P g_{2}\right)_{+}(y, z)$. 
Case 2. $\left|f_{z}(y, z)\right|>\tau \varepsilon$. Then by definition $\left(P g_{1}\right)_{+}(y, z)=\left(P g_{2}\right)_{+}(y, z)=0$. Hence $P g_{1} \sim$ $\mathrm{Pg}_{2}$.

The proof of (ii) follows immediately from Proposition A1.4 since for all $\tilde{g}, \tilde{g}^{\prime} \in \tilde{E}_{\varepsilon}(1)$ :

$$
\begin{gathered}
\left\|\tilde{P} \tilde{g}-\tilde{P} \tilde{g}^{\prime}\right\|_{\varepsilon}=\left\|\widetilde{P g}-\widetilde{P g^{\prime}}\right\|_{\varepsilon}=\left\|P g-P g^{\prime}\right\|_{\varepsilon} \\
\leq k\left\|g-g^{\prime}\right\|_{\varepsilon}=k\left\|\tilde{g}-\tilde{g}^{\prime}\right\|_{\varepsilon} .
\end{gathered}
$$

Continuation of the proof of lemma A1. From Proposition A1.5 we infer the existence of a unique $\tilde{\boldsymbol{g}} \in \tilde{E}_{\varepsilon}(1)$ with $\boldsymbol{P} \tilde{g}=\tilde{g}$. This means: for all $(y, z) \in$ $\mathbb{R}^{n} \times[-\tau \varepsilon, \tau \varepsilon]: P g(y, z)=g(y, z)$, or equivalently (as can be checked from the definition of $P$ ):

$$
\left.(1+g) \circ(L+\lambda)\right|_{f^{-1}\left(\mathbb{R}^{n} \times[-\tau \varepsilon, \tau \varepsilon]\right)}=\left.\left(L+\lambda^{\prime}\right) \circ(1+g)\right|_{f^{-1}\left(\mathbf{R}^{n} \times[-\tau \varepsilon, \tau \varepsilon]\right)} .
$$

So there is a unique class $\tilde{g} \in \tilde{E}_{\varepsilon}(1)$ with the above property. On the other hand it follows from the estimates in Proposition A1.1 that the conjugacy equation (1+ $g) \circ f=f^{\prime} \circ(1+g)$ fixes the definition of $g$ on the whole $\mathbb{R}^{n} \times \mathbb{P}$. This implies the existence and the unicity of the $g$ in Lemma $A 1$, and finishes the proof.

LEMMA A2. (We use the symbols of Lemma A1.) If we take $\delta<\delta_{0}$ sufficiently close to $\delta_{0}$ and if we take $\varepsilon$ of Lemma A1 small then $h$ is a homeomorphism.

Proof. First we apply Lemma A1 for $\delta_{0}$ and obtain an $\varepsilon_{0}>0$ such that for each $\lambda$, $\lambda^{\prime} \in A\left(\varepsilon_{0}, C\right)$ there exists a unique mapping

such that

$$
g_{0}=\left(g_{0-}, g_{0+}, g_{0 z}\right): \mathbb{R}^{n} \times \mathbb{R} \rightarrow \mathbb{R}^{n-} \times \mathbb{R}^{n+} \times \mathbb{R}
$$

$$
\left(1+g_{0}\right) \circ(L+\lambda)=\left(L+\lambda^{\prime}\right) \circ\left(1+g_{0}\right)
$$

and which satisfies:

$$
\begin{array}{ll}
g_{0} \text { is uniformly continuous on } \mathbb{R}^{n} \times\left[-\tau \varepsilon_{0}, \tau \varepsilon_{0}\right], & (\mathrm{A} 1.10)(\mathrm{a}) \\
g_{0+}=0 \text { outside }|z| \leq \tau \varepsilon_{0}, & (\mathrm{~A} 1.10)(\mathrm{b}) \\
\left|\frac{g_{0-}(y, z)}{z^{1-\delta_{0}}}\right| \leq 1 \text { and }\left|\frac{g_{0+}(y, z)}{z^{1-\delta_{0}}}\right| \leq 1 & (\mathrm{~A} 1.10)(\mathrm{c}) \\
\mid \frac{g_{0 z}(y, z)}{z^{2-2 \delta_{0}} \mid \leq 1 .} & \text { for all }(y, z) \in \mathbb{R}^{n} \times\left[-\tau \varepsilon_{0}, \tau \varepsilon_{0}\right],
\end{array}
$$

Choose $\varepsilon_{0}>0$ such that $a \tau \varepsilon_{0}<1$. Now let $\delta<\delta_{0}$ be close to $\delta_{0}$ such that

and

$$
\left\|L_{-}\right\|<a^{1-\delta}
$$

$$
\left\|L_{+}^{-1}\right\| a^{1-\delta}<1
$$

Fix $\delta$.

We apply Lemma $A 1$ for this $\delta$ and infer the existence of an $\varepsilon>0$ such that for each $\lambda, \lambda^{\prime} \in A(\varepsilon, C)$ there exists a unique mapping $g=\left(g_{-}, g_{+}, g_{z}\right)$ such that $(1+$ $g) \circ(L+\lambda)=\left(L+\lambda^{\prime}\right) \circ(1+g)$ and which satisfies

$$
\begin{aligned}
& g \text { is uniformly continuous on } \mathbb{R}^{n} \times[-\tau \varepsilon, \tau \varepsilon], \\
& g_{+}=0 \text { outside }|z| \leq \tau \varepsilon,
\end{aligned}
$$




$$
\begin{aligned}
& \left|\frac{g_{-}(y, z)}{z^{1-\delta}}\right| \leq 1 \text { and }\left|\frac{g_{+}(y, z)}{z^{1-\delta}}\right| \leq 1 \\
& \left|\frac{g_{z}(y, z)}{z^{2-2 \delta}}\right| \leq 1 .
\end{aligned}
$$

for all $(y, z) \in \mathbb{R}^{n} \times[-\tau \varepsilon, \tau \varepsilon]$

Moreover we can take care that $\varepsilon \leq \varepsilon_{0}\left(1-\left(a \tau \varepsilon_{0}\right)^{1-2 \delta}\right)$. Fix arbitrary $\lambda, \lambda^{\prime} \in A(\varepsilon, C)$.

By interchanging $\lambda$ and $\lambda^{\prime}$ we also infer the existence of a unique mapping $\tilde{g}$ such that $(1+\tilde{g}) \circ\left(L+\lambda^{\prime}\right)=(L+\lambda) \circ(1+\tilde{g})$ and with similar properties as in (A1.11). Combining these two we can write:

$$
\begin{aligned}
(1+\tilde{g}) \circ(1+g) \circ(L+\lambda) & =(1+\tilde{g}) \circ\left(L+\lambda^{\prime}\right) \circ(1+g) \\
& =(L+\lambda) \circ(1+\tilde{g}) \circ(1+g) .
\end{aligned}
$$

In this we have:

$$
\begin{aligned}
(1+\tilde{g}) \circ(1+g) & =1+g+\tilde{g} \circ(1+g) \\
& =1+\tilde{\tilde{g}}
\end{aligned}
$$

where $\tilde{\tilde{g}}=\mathrm{g}+\tilde{\mathrm{g}} \circ(1+\mathrm{g})$.

Observe that $A(\varepsilon, C) \subset A\left(\varepsilon_{0}, C\right)$, so $\lambda \in A\left(\varepsilon_{0}, C\right)$. We want to show that $\tilde{\tilde{g}}$ satisfies the properties in (A1.10); then uniqueness implies $\tilde{g}=0$, hence $(1+\tilde{g}) \circ(1+g)=1$; in a similar way also $(1+g) \circ(1+\tilde{g})=1$; so $1+g$ has an inverse namely $1+\tilde{g}$.

So let us check (A1.10) for $\tilde{g}$. From the estimates on $f_{z}$ in Proposition A1.1 it immediately follows from the conjugacy equation that $\tilde{g}$ is uniformly continuous on $\mathbb{R}^{n} \times\left[-\tau \varepsilon_{0}, \tau \varepsilon_{0}\right]$.

First we look at how (A1.11)(d) implies an estimate for $g_{z}$ on $\mathbb{R}^{\mathrm{n}} \times \mathbb{R}$. Let $(y, z) \in$ $\mathbb{R}^{n} \times \mathbb{R},|z| \geq \tau \varepsilon$. There exists an $N \in \mathbb{N}$ such that $|z| \in\left[a^{N} \tau \varepsilon, a^{N+1} \tau \varepsilon\right]$. As $\operatorname{supp}(\lambda) \cup$ $\operatorname{supp}\left(\lambda^{\prime}\right) \subset \mathbb{R}^{n} \times[-\tau \varepsilon, \tau \varepsilon]$ it follows from the conjugacy equation that

$$
g_{z}(y, z)=a^{N+1} g_{z}\left(L^{-N-1}(y, z)\right),
$$

where $L^{-N-1}(y, z) \in \mathbb{R}^{n} \times[-\tau \varepsilon, \tau \varepsilon]$. Denote $\left(y^{\prime}, z^{\prime}\right)=L^{-N-1}(y, z) ;$ then $z=a^{N+1} z^{\prime}$ and

$$
\begin{aligned}
\frac{\left|g_{z}(y, z)\right|}{|z|^{2-2 \delta}} & =\frac{\left|a^{N+1} g_{z}\left(y^{\prime}, z^{\prime}\right)\right|}{\left|z^{\prime}\right|^{2-2 \delta}}\left|\frac{z^{\prime}}{z}\right|^{2-2 \delta} \\
& \leq a^{N+1}\left(\frac{1}{a^{N+1}}\right)^{2-2 \delta}=\left(\frac{1}{a^{1-2 \delta}}\right)^{N+1} \\
& \leq 1
\end{aligned}
$$

since $\left.\delta \in] 0, \frac{1}{2}\right]$.

We conclude that for all $(y, z) \in \mathbb{R}^{n} \times \mathbb{R} \cdot\left|g_{z}(y, z)\right| /|z|^{2-2 \delta} \leq 1$. The same holds for g. We prove $(\mathrm{A} .1 .10)(\mathrm{b})$ for $\tilde{\tilde{g}}$. So let $|z| \geq \tau \varepsilon_{0}$.

We first show the implication $|z| \in\left[\tau \varepsilon_{0}, a \tau \varepsilon_{0}\right] \Rightarrow\left|z+g_{z}(y, z)\right| \geq \tau \varepsilon$. Let $|z| \epsilon$ $\left[\tau \varepsilon_{0}, a \tau \varepsilon_{0}\right]$.

Then

$$
\left|g_{z}(y, z)\right| \leq|z|^{2-2 \delta} \leq\left(a \tau \varepsilon_{0}\right)^{2-2 \delta}
$$


so

$$
\begin{aligned}
\left|z+g_{z}(y, z)\right| & \geq|z|\left(1-|z|^{1-2 \delta}\right) \\
& \geq \tau \varepsilon_{0}\left(1-\left(a \tau \varepsilon_{0}\right)^{1-2 \delta}\right) \\
& \geq \tau \varepsilon .
\end{aligned}
$$

Now let $N \in \mathbb{N}$ and suppose by induction that the following implication holds for all $(y, z) \in \mathbb{R}^{n} \times \mathbb{R}$ :

$$
|z| \in\left[a^{N} \tau \varepsilon_{0}, a^{N+1} \tau \varepsilon_{0}\right] \Rightarrow\left|z+g_{z}(y, z)\right| \geq a^{N} \tau \varepsilon .
$$

Suppose $|z| \in\left[a^{N+1} \tau \varepsilon_{0}, a^{N+2} \tau \varepsilon_{0}\right]$. Then, by the conjugacy equation we have:

since $\left|\left(L^{-1}(y, z)\right)_{z}\right| \in\left[a^{N} \tau \varepsilon_{0}, a^{N+1} \tau \varepsilon_{0}\right]$.

$$
\begin{aligned}
\left|z+g_{z}(y, z)\right| & =\left|a(1+g)_{z}\left(L^{-1}(y, z)\right)\right| \\
& \geq a a^{N} \tau \varepsilon=a^{N+1} \tau \varepsilon
\end{aligned}
$$

We can conclude that for all $(y, z) \in \mathbb{R}^{n} \times \mathbb{R}$ :

$$
|z| \geq \tau \varepsilon_{0} \Rightarrow\left|z+g_{z}(y, z)\right| \geq \tau \varepsilon .
$$

Since $\tilde{g}_{+}=0$ outside $|z| \leq \tau \varepsilon$ and since $g_{+}=0$ outside $|z| \leq \tau \varepsilon$ we see from the formula for $\tilde{\tilde{g}}$ that $\tilde{g}_{+}=0$ outside $|z| \leq \tau \varepsilon_{0}$.

We prove $(\mathrm{A1} 10)(\mathrm{c})$ for $\tilde{\tilde{g}}$. Remember that the supports of $\lambda, \lambda^{\prime}$ lie in $\mathbb{R}^{n} \times[-\tau \varepsilon, \tau \varepsilon]$.

Let $(y, z) \in \mathbb{R}^{n} \times \mathbb{R},|z| \geq \tau \varepsilon$. There exists an $N \in \mathbb{N}$ such that $|z| \in\left[a^{N} \tau \varepsilon, a^{N+1} \tau \varepsilon\right]$. We have from the conjugacy equation:

$$
g_{-}(y, z)=L_{-}^{N+1} g_{-}\left(L^{-N-1}(y, z)\right) .
$$

Denote $\left(y^{\prime}, z^{\prime}\right)=L^{-N-1}(y, z)$; then $z=a^{N+1} z^{\prime}$ and

$$
\begin{aligned}
\frac{\left|g_{-}(y, z)\right|}{|z|^{1-\delta}} & \leq\left\|L_{-}\right\|^{N+1} \frac{\left|g_{-}\left(y^{\prime}, z^{\prime}\right)\right|}{\left|z^{\prime}\right|^{1-\delta}}\left|\frac{z^{\prime}}{z}\right|^{1-\delta} \\
& \leq\left(\frac{\left\|L_{-}\right\|}{a^{1-\delta}}\right)^{N+1} .
\end{aligned}
$$

So we may conclude that for all

Similarly for $\tilde{\boldsymbol{g}}_{-}$.

$$
(y, z) \in \mathbb{R}^{n} \times \mathbb{R}: \frac{\left|g_{-}(y, z)\right|}{|z|^{1-\delta}} \leq 1 .
$$

Furthermore, by (A1.11)(b), for all

The same holds for $\tilde{\mathbf{g}}_{+}$.

$$
(y, z) \in \mathbb{R}^{n} \times \mathbb{R}:\left|\frac{g_{+}(y, z)}{z^{1-\delta}}\right| \leq 1 .
$$

So we can write for all $(y, z) \in \mathbb{R}^{n} \times\left[-\tau \varepsilon_{0}, \tau \varepsilon_{0}\right]$ :

$$
\begin{aligned}
\frac{\left|\tilde{g}_{-}(y, z)\right|}{|z|^{1-\delta_{0}}} & \leq \frac{\left|g_{-}(y, z)\right|}{|z|^{1-\delta_{0}}}+\frac{\left|\tilde{g}_{-}((1+g)(y, z))\right|}{|z|^{1-\delta_{0}}} \\
& \leq \frac{\left|g_{-}(y, z)\right|}{|z|^{1-\delta}} \cdot \frac{|z|^{1-\delta}}{|z|^{1-\delta_{0}}}+\frac{\left|z+g_{z}(y, z)\right|^{1-\delta}}{|z|^{1-\delta_{0}}} \\
& \leq|z|^{\delta_{0}-\delta}+\frac{\left.\left.|z|^{1-\delta}|1+| z\right|^{1-2 \delta}\right|^{1-\delta}}{|z|^{1-\delta_{0}}} \\
& \leq|z|^{\delta_{0}-\delta}+|z|^{\delta_{0}-\delta}\left(1+|z|^{1-2 \delta}\right)^{1-\delta} \\
& \leq\left|\tau \varepsilon_{0}\right|^{\delta_{0}-\delta}+\left|\tau \varepsilon_{0}\right|^{\delta_{0}-\delta}\left(1+\left|\tau \varepsilon_{0}\right|^{1-2 \delta}\right)
\end{aligned}
$$


If $\varepsilon_{0}$ is small (only depending on $\tau, \delta_{0}, \delta$ ) we can make this less than 1. A similar argument works for $\tilde{\boldsymbol{g}}_{+}$. We finally prove $(\mathrm{A} 1.10)(\mathrm{d})$ for $\tilde{\tilde{g}}$ :

$$
\begin{aligned}
\frac{\left|\tilde{g}_{z}(y, z)\right|}{|z|^{2-2 \delta_{0}}} & \leq \frac{\left|g_{z}(y, z)\right|}{|z|^{2-2 \delta_{0}}}+\frac{\left|\tilde{g}_{z}((1+g)(y, z))\right|}{|z|^{2-2 \delta_{0}}} \\
& \leq|z|^{2 \delta_{0}-2 \delta}+|z|^{2 \delta_{0}-2 \delta}\left(1+|z|^{1-2 \delta}\right)^{1-\delta} \\
& \leq\left|\tau \varepsilon_{0}\right|^{2 \delta_{0}-2 \delta}+\left|\tau \varepsilon_{0}\right|^{2 \delta_{0}-2 \delta}\left(1+\left|\tau \varepsilon_{0}\right|^{1-2 \delta}\right)^{1-\delta} \\
& \leq 1,
\end{aligned}
$$

if $\varepsilon_{0}$ is small enough.

A2. Theorem $A$ reduces to Lemma 2. As before we have two diffeomorphisms $f, f^{\prime}$ (or local diffeomorphisms) on $\mathbb{R}^{n} \times\{0\}$, with

$$
\begin{aligned}
& f\left|\mathbb{R}^{n} \times\{0\}=f^{\prime}\right| \mathbb{R}^{n} \times\{0\} \\
& \gamma(y, 0)=\gamma^{\prime}(y, 0),
\end{aligned}
$$

for $y \in \mathbb{R}^{n}$. It follows that $A=d f(0)$ respectively $A^{\prime}=d f^{\prime}(0)$ is of the form

$$
\begin{aligned}
A & =\left[\begin{array}{ll}
B & * \\
0 & a
\end{array}\right], \text { respectively } \\
A^{\prime} & =\left[\begin{array}{ll}
B^{\prime} & * \\
0 & a^{\prime}
\end{array}\right]
\end{aligned}
$$

with $B=B^{\prime}, a=a^{\prime}$ and $*$ some term which is irrelevant. Using a linear coordinate change respecting $\mathbb{R}^{n} \times\{0\}$, we can put $B=B^{\prime}$ in the form

$$
\boldsymbol{B}=\left[\begin{array}{cc}
\boldsymbol{B}_{-} & 0 \\
0 & \boldsymbol{B}_{+}
\end{array}\right],
$$

where the eigenvalues of $B_{-}$are less than $|a|$, (in norm), and the norms of the eigenvalues of $B_{+}$are bigger or equal to $|a|$. Denoting $\|\cdot\|$ for the operator norm of a linear map and choosing $\delta \in] 0, \frac{1}{2}\left[\right.$ such that $|a|^{1-\delta}$ is not an eigenvalue of $B$, we can take this linear coordinate change so that

$$
\begin{aligned}
& \left\|B_{-}\right\|<|a|^{1-\delta}, \\
& \left\|B_{+}^{-1}\right\| \cdot|a|^{1-\delta}<1 .
\end{aligned}
$$

So, using this coordinate change, we can assume that $d f(0), d f^{\prime}(0)$ are of the form

$$
\begin{aligned}
& d f(0)=\left[\begin{array}{ccc}
L_{-} & 0 & b_{-} \\
0 & L_{+} & b_{+} \\
0 & 0 & a
\end{array}\right], \\
& d f^{\prime}(0)=\left[\begin{array}{ccc}
L_{-} & 0 & b_{-}^{\prime} \\
0 & L_{+} & b_{+}^{\prime} \\
0 & 0 & a
\end{array}\right] .
\end{aligned}
$$

Write

$$
L=\left[\begin{array}{ccc}
L_{-} & 0 & 0 \\
0 & L_{+} & 0 \\
0 & 0 & a
\end{array}\right]
$$


The $f$ and $f$ are of the form

$$
\begin{aligned}
& f(x)=L x+\left(\begin{array}{c}
b_{-} \\
b_{+} \\
0
\end{array}\right) z+P(x), \\
& f^{\prime}(x)=L x+\left(\begin{array}{c}
b_{-}^{\prime} \\
b_{+}^{\prime} \\
0
\end{array}\right) z+P^{\prime}(x),
\end{aligned}
$$

where $|P(x)|,\left|P^{\prime}(x)\right|=O\left(|x|^{2}\right)$.

Now we will globalize the local diffeomorphisms $f$ and $f^{\prime}$. Take a bump function $\beta$ such that

(1) $\beta: \mathbb{R} \rightarrow[0,1]$ is $C^{\infty}$

(2) $\beta(z)=0$ outside $|z| \geq 1$

(3) $\beta(z)=1$ for $|z| \leq \frac{1}{2}$.

We define for $\varepsilon>0$

$$
\lambda_{\varepsilon}(x)=\beta(|x|) \beta\left(\frac{|z|}{\varepsilon}\right)\left(\begin{array}{c}
b_{-} \\
b_{+} \\
0
\end{array}\right) z+\beta\left(\frac{|x|}{\varepsilon}\right) P(x)
$$

and $f_{\varepsilon}=L+\lambda_{\varepsilon} ;$ similarly for $\lambda_{\varepsilon}^{\prime}$ and $f_{\varepsilon}^{\prime}=L+\lambda_{\varepsilon}^{\prime}$.

Clearly $f_{\varepsilon}\left|\mathbb{R}^{n} \times\{0\}=f_{\varepsilon}^{\prime}\right| \mathbb{R} \times\{0\}$. We check the desired properties for $\lambda_{\varepsilon}$ (those for $\lambda_{\varepsilon}^{\prime}$ are the same). The support of $\lambda_{\varepsilon}$ is compact and lies in $\mathbb{R}^{n} \times[-\varepsilon, \varepsilon]$. We can choose $\varepsilon_{0}>0$ and $M>0$ such that for all $x \in \mathbb{R}^{n} \times \mathbb{R}$ with $|x|<\varepsilon_{0}:\|D P(x)\| \leq M|x|$ and $|P(x)| \leq M|x|^{2}$.

If $0<\varepsilon \leq \varepsilon_{0}$ then $\left|\lambda_{\varepsilon}\right| \leq C \varepsilon$ with $C>0$ not depending on $\varepsilon$. Denote $l=\left|\left(b_{-}, b_{+}, 0\right)\right|$; then the Leibniz formula yields the following, for $0<\varepsilon \leq \varepsilon_{0}$ : if $|x|<\varepsilon$ then

$$
\operatorname{Lip}\left(\beta\left(\frac{|x|}{\varepsilon}\right) P(x)\right) \leq \frac{1}{\varepsilon} \operatorname{Lip} \beta \cdot M \varepsilon^{2}+M \varepsilon ;
$$

as $\beta(|x| / \varepsilon) P(x)=0$ for $|x| \geq \varepsilon$ this estimate remains correct on $\mathbb{R}^{n} \times \mathbb{R}$; for $|z|<\varepsilon$ :

$$
\operatorname{Lip}\left(\beta(|x|) \beta\left(\frac{|z|}{\varepsilon}\right)\left(\begin{array}{c}
b_{-} \\
b_{+} \\
0
\end{array}\right) z\right) \leq \operatorname{Lip} \beta \cdot l \varepsilon+\frac{1}{\varepsilon} \operatorname{Lip} \beta \cdot l \varepsilon+l,
$$

which remains correct on $\mathbb{R}^{n} \times \mathbb{R}$ since

$$
\beta(|x|) \beta\left(\frac{|z|}{\varepsilon}\right)\left(\begin{array}{c}
b_{-} \\
b_{+} \\
0
\end{array}\right) z=0 \quad \text { for }|z| \geqq \varepsilon .
$$

Hence $\operatorname{Lip}\left(\lambda_{\varepsilon}\right)$ is bounded by some $C>0$ not depending on $\varepsilon\left(\leq \varepsilon_{0}\right)$. Similarly we can estimate $\operatorname{Lip}_{y} \lambda_{\varepsilon}$, meaning the Lipschitz constant respecting the $y$-variable:

$$
\operatorname{Lip}_{y}\left(\beta(|x|) \beta\left(\frac{|z|}{\varepsilon}\right)\left(\begin{array}{c}
b_{-} \\
b_{+} \\
0
\end{array}\right) z\right) \leq \operatorname{Lip} \beta \cdot l \varepsilon
$$


and

$$
\operatorname{Lip}_{y}\left(\beta\left(\frac{|x|}{\varepsilon}\right) P(x)\right) \leq \frac{1}{\varepsilon} \operatorname{Lip} \beta \cdot M \varepsilon^{2}+M \varepsilon ;
$$

hence $\operatorname{Lip}_{y}\left(\lambda_{\varepsilon}\right) \leq C \varepsilon$ for some $C>0$ independent of $\varepsilon\left(\leq \varepsilon_{0}\right)$. The $C^{1}$ function $\gamma_{\varepsilon}$ is defined by

$$
\lambda_{\varepsilon, z}(y, z)=\gamma_{\varepsilon}(y, z) \cdot z
$$

where $\lambda_{\varepsilon, z}$ is the $z$-component of $\lambda_{\varepsilon}$. Since $P_{z}(y, 0)=0$ one can write $P_{z}(y, z)=$ $q(y, z) \cdot z$ for a $C^{1}$ function $q$. By definition

$$
\gamma_{\varepsilon}(x)=\beta\left(\frac{|x|}{\varepsilon}\right) q(x)
$$

As $q(y, 0)=\left(\partial P_{z} / \partial z\right)(y, 0)$ we have $q(0,0)=0$ and for $\varepsilon_{0}>0$ sufficiently small one can find $N>0$ so that for $|x|<\varepsilon_{0}:|q(x)| \leq N|x|$. Consequently, for $0<\varepsilon \leq \varepsilon_{0}$ we have (taking into account that $\gamma_{\varepsilon}(x)=0$ for $|x| \geq \varepsilon$ ):

$$
\left|\gamma_{\varepsilon}(x)\right| \leq C \varepsilon
$$

and

$$
\operatorname{Lip}\left(\gamma_{\varepsilon}\right) \leq \frac{1}{\varepsilon} \operatorname{Lip} \beta \cdot N \varepsilon+\operatorname{Lip} q \leq C,
$$

for some $C>0$ not depending on $\varepsilon$.

All the conditions of Lemmas $\mathrm{A} 1$ and $\mathrm{A} 2$ are satisfied. As $f$ and $f_{\varepsilon}$ coincide on a small neighbourhood of 0 , it follows that Theorem $A$ reduces to Lemma A2.

Remark. In case $f$ and $f^{\prime}$ are the time-1-mappings of the resp. flows $\left(X_{t}\right)_{t \in \mathbb{R}}$ and $\left(X_{t}^{\prime}\right)_{t \in \mathbf{R}}$ we can use the same method as in $[\mathbf{P u}]$ to show that $\left(X_{t}\right)$ and $\left(X_{t}^{\prime}\right)$ are conjugate in the neighbourhood of 0 .

Using a bumpfunction we extend $\left(X_{t}\right)$ and $\left(X_{t}^{\prime}\right)$ to flows which are globally defined. Let us still denote them by $\left(X_{t}\right)$ and $\left(X_{t}^{\prime}\right)$. If $f$ and $f^{\prime}$ are their respective time 1-mappings and $h$ is the unique conjugacy as given by Theorem $\mathrm{A}$, then one shows that $h=\int_{0}^{1}\left(X_{t}^{\prime} \circ h \circ X_{-t}\right) d t$, and hence that $h$ serves as a conjugacy between $X_{t}$ and $X_{t}^{\prime}$ for any $t$.

Appendix 2. Characterization of the conjugacies in theorem $A$, in the special case of an $(n, 1)$-saddle

In this Appendix we work again with diffeomorphisms $f$ and $f^{\prime}$ as in Theorem A of Appendix 1. However, $f\left|\mathbb{R}^{n} \times\{0\}=f^{\prime}\right| \mathbb{R}^{n} \times\{0\}=g$ is supposed to be a contraction (not necessarily hyperbolic) and moreover $f$ is the 'normal linearization' of $f^{\prime}$ along $\mathbb{R}^{n} \times\{0\}$. We will show that any conjugacy between $f$ and $f^{\prime}$ given in the neighbourhood of a fundamental domain of $g$ and 'tangent to the identity along $\mathbb{R}^{n} \times\{0\}$ ' (like in Theorem $\mathrm{A}$ ) extends in a unique way to a conjugacy between $f$ and $f^{\prime}$.

Let $f^{\prime}: \mathbb{R}^{n} \times \mathbb{R} \rightarrow \mathbb{R}^{n} \times \mathbb{R}:(y, z) \rightarrow\left(f_{y}^{\prime}(y, z), f_{z}^{\prime}(y, z)\right)$ be of class $C^{2}$ and defined on an open neighbourhood $V^{\prime}$ of 0 and a diffeomorphism onto its image, with $f^{\prime}(0)=0$. Suppose that $f_{z}^{\prime}(y, z)=\gamma^{\prime}(y, z) \cdot z$ where $\gamma^{\prime}$ is $C^{1}$ and $\gamma^{\prime}(0,0)=a>1$. Let $f$ be the 
'normal linearization' of $f^{\prime}$, i.e. $f: \mathbb{R}^{n} \times \mathbb{R} \rightarrow \mathbb{R}^{n} \times \mathbb{R}:(y, z) \rightarrow(g(y), \gamma(y) z)$ with $g(y)=$ $f_{y}^{\prime}(y, 0)$ and $\gamma(y)=\gamma^{\prime}(y, 0)$. Assume $\left.V=V_{1} \times\right]-\varepsilon, \varepsilon\left[\right.$ where $V_{1}$ is so small that for all $y \in V_{1}: \gamma(y)>b>1$.

Let us moreover suppose that $g$ is a local contraction in 0 in the sense that for some Riemannian metric on $\mathbb{R}^{n}: V_{1} \supset \bar{B}_{1}(0)$ (the closed unit ball), $g\left(\bar{B}_{1}(0)\right) \subset B_{1}(0)$ and $\bigcap_{n \geq 0} g^{n}\left(\bar{B}_{1}(0)\right)=\{0\}$.

THEOREM B. Let $f, f^{\prime}$ be as above and let h be any mapping, homeomorphism onto its image, defined on some neighbourhood $W$ of $\left(\bar{B}_{1}(0) \backslash g\left(B_{1}(0)\right)\right) \times\{0\}$ in $\mathbb{R}^{n} \times \mathbb{R}$, $W \subset V$, with the property $h \circ f=f^{\prime} \circ h, h=\left(h_{y}, h_{z}\right)$,

$$
\left|\frac{h_{z}-z}{z^{1+\alpha}}\right| \leq 1, \quad\left|\frac{h_{y}-y}{z^{\alpha}}\right| \leq 1 .
$$

Then h extends uniquely to a $C^{0}$ conjugacy between $f$ and $f^{\prime}$ on a neighbourhood of 0 . Proof. Because of Theorem A, $f$ is $C^{0}$ conjugate to $f^{\prime}$ around the origin; moreover the conjugacy $\bar{H}=\left(\bar{H}_{y}, \bar{H}_{z}\right)$ is such that

and

$$
\left|\frac{\bar{H}_{z}-z}{z^{1+\alpha}}\right| \leq 1
$$

$$
\left|\frac{\bar{H}_{y}-y}{z^{\alpha}}\right| \leq 1
$$

for some $\alpha>0$. We take care that $f \circ \bar{H}=\bar{H} \circ f^{\prime}$ holds on $V$. Taking $V^{\prime} \circ V$ sufficiently small and extending $h$ by means of the condition $h \circ f=f^{\prime} \circ h$ ('saturation'); we may suppose that $h$ is defined on $W \supset V^{\prime} \backslash(\{0\} \times \mathbb{R})$ and that $h(W) \subset V$. Consider $K=$ $\bar{H} \circ h: W \rightarrow \mathbb{R}^{n} \backslash(\{0\} \times \mathbb{R}) ; K$ is a homeomorphism onto its image, $K \circ f=f \circ K$ and $K=\left(K_{y}, K_{z}\right)=(y+P, z+Q)$ with

$$
\left|\frac{P}{z^{\alpha}}\right| \leq 1, \quad\left|\frac{Q}{z^{1+\alpha}}\right| \leq 1 .
$$

We also define $K$ on $\{0\} \times \mathbb{R}$ to be the identity; we shall prove that $K$ is continuous. As a consequence $H=\bar{H}^{-1} \circ K$ is a $C^{0}$ conjugacy between $f$ and $f^{\prime}$ in the neighbourhood of 0 , with $H \mid W=h$. It is clearly the unique conjugacy extending $h$.

So take a sequence $\left(y_{i}, z_{i}\right) \rightarrow(0, z), i \rightarrow \infty, y_{i} \neq 0$. For each $i \in \mathbb{N}$ consider $N_{i} \in \mathbb{N}$ with $g^{-N_{i}}\left(y_{i}\right) \in \widetilde{B}_{1}(0) \backslash g\left(B_{1}(0)\right)$. Remember: $f(y, z)=(g(y), \gamma(y) z)$.

Clearly $N_{i} \rightarrow \infty$ for $i \rightarrow \infty$. As $K$ commutes with $f$ we have that $K\left(y_{i}, z_{i}\right)=$ $f^{N_{i}} \circ K \circ f^{-N_{i}}\left(y_{i}, z_{i}\right)$.

More explicitly:

with $\bar{y}_{i} \in \vec{B}_{1}(0) \backslash g\left(B_{1}(0)\right)$,

$$
f^{N_{i}}\left(y_{i}, z_{i}\right)=\left(g^{-N_{i}}\left(y_{i}\right), \frac{z_{i}}{\prod_{j=1}^{N_{i}} \gamma\left(g^{-j}\left(y_{i}\right)\right)}\right)=\left(\bar{y}_{i}, \bar{z}_{i}\right)
$$

$$
K\left(\bar{y}_{i}, z_{i}\right)=\left(\bar{y}_{i}+P\left(\bar{y}_{i}, \bar{z}_{i}\right), \bar{z}_{i}+Q\left(\bar{y}_{i}, \bar{z}_{i}\right)\right)=\left(\bar{y}_{i}, \bar{z}_{i}\right)
$$

and

$$
K\left(y_{i}, z_{i}\right)=\left(g^{N_{i}}\left(\tilde{y}_{i}\right),\left(\prod_{j=0}^{N_{i}-1} \gamma\left(g^{j}\left(\tilde{y}_{i}\right)\right)\right) z_{i}\right)
$$


Take $I \in \mathbb{N}$ large enough so that for all $i \geq I: g\left(\bar{y}_{i}\right) \in \bar{B}_{1}(0)$; this is possible because $\gamma(y)>b>1$, because $K \mid \mathbb{R}^{n} \times\{0\}$ is the identity and because $K$ is continuous on $W$. It follows that $g^{N_{i}}\left(\overline{\bar{y}}_{i}\right) \rightarrow 0$ for $i \rightarrow \infty$.

Now

$$
K_{z}\left(y_{i}, z_{i}\right)=\left(\prod_{j=0}^{N_{i}-1} \gamma\left(g^{j}\left(\overline{\bar{y}}_{i}\right)\right)\right)\left[\frac{z_{i}}{\prod_{j=1}^{N_{i}} \gamma\left(g^{-j}\left(y_{i}\right)\right)}+Q\left(\bar{y}_{i}, \bar{z}_{i}\right)\right]
$$

and as $\bar{y}_{i}=g^{-N_{i}}\left(y_{i}\right)$ we get

$$
K_{z}\left(y_{i}, z_{i}\right)=\left(\prod_{j=0}^{N_{i}-1} \frac{\gamma\left(g^{j}\left(\overline{\bar{y}}_{i}\right)\right)}{\gamma\left(g^{j}\left(\bar{y}_{i}\right)\right)}\right) z_{i}+\left(\prod_{j=0}^{N_{i}-1} \gamma\left(g^{j}\left(\overline{\bar{y}}_{i}\right)\right)\right) Q\left(\bar{y}_{i}, \bar{z}_{i}\right) .
$$

Furthermore

$$
\begin{aligned}
\left|Q\left(\bar{y}_{i}, \bar{z}_{i}\right)\right| & \leq\left|\bar{z}_{i}\right|^{1+\alpha} \\
& =\left|\frac{z_{i}}{\prod_{j=1}^{N_{i}} \gamma\left(g^{-j}\left(y_{i}\right)\right)}\right|^{1+\alpha} \\
& =\left|\frac{z_{i}}{\prod_{j=0}^{N_{i}-1} \gamma\left(g^{j}\left(\bar{y}_{i}\right)\right)}\right|^{1+\alpha}
\end{aligned}
$$

It suffices to show that

$$
\lim _{i \rightarrow \infty} \prod_{j=0}^{N_{i}-1} \frac{\gamma\left(g^{j}\left(\bar{y}_{i}\right)\right)}{\gamma\left(g^{j}\left(\bar{y}_{i}\right)\right)}=1
$$

because then,

$$
\lim _{i \rightarrow \infty}\left(\prod_{j=0}^{N_{i}-1} \gamma\left(g^{j}\left(\bar{y}_{i}\right)\right)\right) \cdot\left(\frac{z_{i}}{\prod_{j=0}^{N_{i}-1} \gamma\left(g^{j}\left(\bar{y}_{i}\right)\right)}\right)^{1+\alpha}=0
$$

for $\alpha>0$ which implies $\lim _{i \rightarrow \infty} K\left(y_{i}, z_{i}\right)=(0, z)$.

As $\gamma$ is $C^{1}$ there exists a $C_{1}>0$ such that $\gamma(y)=\gamma\left(y^{\prime}\right)+L\left(y, y^{\prime}\right)$ with $\left|L\left(y, y^{\prime}\right)\right| \leqq$ $C_{1}\left|y-y^{\prime}\right|$. From this:

$$
\frac{\gamma\left(g^{j}\left(\bar{y}_{i}\right)\right)}{\gamma\left(g^{j}\left(\bar{y}_{i}\right)\right)}=1+\frac{L\left(g^{j}\left(\bar{y}_{i}\right), g^{j}\left(\bar{y}_{i}\right)\right)}{\gamma\left(g^{j}\left(\bar{y}_{i}\right)\right)}
$$

and

$$
\begin{aligned}
1-\frac{C_{1}}{b}\left|g^{j}\left(\bar{y}_{i}\right)-g^{j}\left(\bar{y}_{i}\right)\right| & \leq 1+\frac{L\left(g^{j}\left(\bar{y}_{i}\right), g^{j}\left(\bar{y}_{i}\right)\right)}{\gamma\left(g^{j}\left(\bar{y}_{i}\right)\right)} \\
& \leq 1+\frac{C_{1}}{b}\left|g^{j}\left(\bar{y}_{i}\right)-g^{j}\left(\bar{y}_{i}\right)\right| .
\end{aligned}
$$

So using $\|D g(0)\| \leq 1$ and taking $V_{1}$ sufficiently small we can find a positive constant $A<b^{\alpha / 2}$ such that for $\forall \bar{y}_{i}, \bar{y}_{i} \in V_{1}$,

$$
1-\frac{C_{1}}{b} A^{j}\left|\overline{\bar{y}}_{i}-\bar{y}_{i}\right| \leq \frac{\gamma\left(g^{j}\left(\bar{y}_{i}\right)\right)}{\gamma\left(g^{j}\left(\bar{y}_{i}\right)\right)} \leq 1+\frac{C_{1}}{b} A^{j}\left|\bar{y}_{i}-\bar{y}_{i}\right| \text {. }
$$

We use

$$
\begin{aligned}
\left|\bar{y}_{i}-\bar{y}_{i}\right| & =\left|P\left(\bar{y}_{i}, \bar{z}_{i}\right)\right| \leq\left|\bar{z}_{i}\right|^{\alpha}=\left|\bar{z}_{i}\right|^{\alpha / 2} \cdot\left|\bar{z}_{i}\right|^{\alpha / 2} \\
& \leq\left|\frac{z_{i}}{b^{N_{i}}}\right|^{\alpha / 2} \cdot\left|\bar{z}_{i}\right|^{\alpha / 2}
\end{aligned}
$$


and obtain

$$
\begin{aligned}
\prod_{j=0}^{N_{i}-1}\left(1-\frac{C_{1}}{b} A^{j}\left|\frac{z_{i}}{b^{N_{i}}}\right|^{\alpha / 2} \cdot\left|\bar{z}_{i}\right|^{\alpha / 2}\right) & \leqq \prod_{j=0}^{N_{i}-1} \frac{\gamma\left(g^{j}\left(\bar{y}_{i}\right)\right)}{\gamma\left(g^{j}\left(\bar{y}_{i}\right)\right)} \\
& \leqq \prod_{j=0}^{N_{i}-1}\left(1+\frac{C_{1}}{b}\left|\frac{z_{i}}{b^{N_{i}}}\right|^{\alpha / 2} \cdot\left|\bar{z}_{i}\right|^{\alpha / 2}\right)
\end{aligned}
$$

We take the logarithm of these expressions; moreover we have the estimates:

$$
\begin{gathered}
\sum_{j=0}^{N_{i}-1} \log \left(1-\frac{C_{1}}{b} A^{j}\left|\frac{z_{i}}{b^{N_{i}}}\right|^{\alpha / 2}\left|\bar{z}_{i}\right|^{\alpha / 2}\right) \\
\geq-\left(\frac{2 C_{1}}{b^{1+\frac{1}{2} N_{i} \alpha}}\right)\left|z_{i}\right|^{\alpha / 2}\left|\bar{z}_{i}\right|^{\alpha / 2} \sum_{j=0}^{N_{i}-1} A^{j} \\
=\frac{-2 C_{1}\left|z_{i}\right|^{\alpha / 2}\left(1-A^{N_{i}}\right)}{b^{1+\frac{1}{2} N_{i} \alpha}(1-A)}\left|\bar{z}_{i}\right|^{\alpha / 2}
\end{gathered}
$$

and similarly

$$
\begin{aligned}
\sum_{j=0}^{N_{i}-1} \log ( & \left.1+\frac{C_{1}}{b} A^{j}\left|\frac{z_{i}}{b^{N_{i}}}\right|^{\alpha / 2}\left|\bar{z}_{i}\right|^{\alpha / 2}\right) \\
\leq & \frac{C_{1}\left|z_{i}\right|^{\alpha / 2}\left(1-A^{N_{i}}\right)}{\left(b^{1+\frac{1}{2} N_{i}^{\alpha}}\right)(1-A)}\left|\bar{z}_{i}\right|^{\alpha / 2}
\end{aligned}
$$

Hence, as $A<b^{\alpha / 2}$, we can find a constant $C_{2}>0$, independent of $i$, such that

$$
\left|\log \left(\prod_{j=0}^{N_{i}-1} \frac{\gamma\left(g^{j}\left(\bar{y}_{i}\right)\right)}{\gamma\left(g^{j}\left(\bar{y}_{i}\right)\right)}\right)\right| \leq C_{2}\left|\bar{z}_{i}\right|^{\alpha / 2}
$$

Since $\left|\bar{z}_{i}\right|^{\alpha / 2} \leq\left|z_{i} / b^{N_{i}}\right|^{\alpha / 2} \rightarrow 0$ for $i \rightarrow \infty$ the proof is finished.

Remark. If $f$ and $f^{\prime}$ are the time 1-mapping of the flows $X_{t}$ resp. $X_{t}^{\prime}$ and if $h$ in the previous construction is a conjugacy between the flows, then $H$ will be a conjugacy between the flows.

\section{Acknowledgement}

The authors wish to thank the referee for pointing out some gaps in the proof of Theorem A.

\section{REFERENCES}

[C1] M. I. T. Camacho. Geometric properties of homogeneous vector fields of degree two in $\mathbb{R}^{3}$. Trans. Am. Math. Soc. 268 (1981), 79-101.

[C2] M. I. T. Camacho. A contribution to the topological classification of homogeneous vector fields in $\mathbb{R}^{3}$. J. Diff. Eq. 57 (1985), 159-171.

[MS] W. de Melo and S. van Strien. Appendix in: Diffeomorphisms on surfaces with a finite number of moduli. Ergod. Th. \& Dynam. Sys. 7 (1987), 415-462.

[Mo] E. Moise. Geometric Topology in Dimensions 2 and 3 (Springer-Verlag, New York, 1977).

[PM] J. Palis \& W. de Melo. Geometric Theory of Dynamical Systems (Springer-Verlag, New York, 1982).

[Pu] C. C. Pugh. On a theorem of P. Hartman. Amer. J. Math. 91 (1969), 363-367. 
[Sh] D. Shafer. Singularities of gradient vectorfields in $\mathbb{R}^{3}$. J. Diff. Eq. 47 (1983), 317-326.

[Ste] S. Sternberg. On the structure of local homeomorphisms of Euclidean n-space II, Amer. J. Math. 80 (1958), 623-631.

[Str] S. van Strien. Normal hyperbolicity and linearisability. Inventiones Mathematicae 87 (1987), 377-384.

[ST] S. van Strien \& G. Tavares dos Santos. Moduli of stability for germs of homogeneous vectorfields on $R^{3}$. J. Diff. Eq. 69 (1987), 63-84.

[ULL] A. M. Urbina, M. León de la Barra \& G. León de la Barra. Finite determinacy in $\mathbf{R}^{3}$. Preprint. 